\title{
Radiation from ring quasi-arrays
}

\section{Knudsen, $\mathbf{H}$.}

\section{Published in:}

I E E E Transactions on Antennas and Propagation

Link to article, DOI:

10.1109/TAP.1956.1144399

Publication date:

1956

\section{Document Version}

Publisher's PDF, also known as Version of record

Link back to DTU Orbit

Citation (APA):

Knudsen, H. (1956). Radiation from ring quasi-arrays. I E E E Transactions on Antennas and Propagation, 4(3), 452-472. https://doi.org/10.1109/TAP.1956.1144399

\section{General rights}

Copyright and moral rights for the publications made accessible in the public portal are retained by the authors and/or other copyright owners and it is a condition of accessing publications that users recognise and abide by the legal requirements associated with these rights.

- Users may download and print one copy of any publication from the public portal for the purpose of private study or research.

- You may not further distribute the material or use it for any profit-making activity or commercial gain

- You may freely distribute the URL identifying the publication in the public portal

If you believe that this document breaches copyright please contact us providing details, and we will remove access to the work immediately and investigate your claim. 


\section{Radiation from Ring Quasi-Arrays}

\section{H. L. KNUDSEN†}

Summary-The present paper constitutes a summary of investigations of certain antenna systems with rotational symmetry, socalled ring arrays and ring quasi-arrays, which have turned out to be or can be supposed to become of practical importance.

Particular stress has been laid on an investigation of the field radiated from homogenous ring arrays of axial dipoles and homogeneous ring quasi-arrays of tangential and radial dipoles; i.e., systems of respectively axial, tangential, and radial dipoles placed equidistantly along a circle and carrying currents of the same numerical value but with a phase that increases uniformly along the circle.

At first a calculation has been made of the radiated field in the case where the number of elements in the antenna system is infinitely large. After that the influence of the finite number of elements is accounted for by the introduction of correction terms. Subsequently, the radiation resistance and the gain have been calculated in a few simple cases.

The antenna systems described above may display super-gain. On the basis of the theory of super-gain an estimate is made of the smallest permissible radius of these antenna systems.

Further an investigation is made of the field from a directional ring array with a finite number of elements to ascertain in particular the influence on the field of the finite number of elements.

\section{INTRODUCTION}

$\mathrm{T}$ HE PURPOSE of a transmitting antenna is to radiate the electromagnetic energy from the transmitter in the required manner in the various directions of space. The radiation diagram aimed at is often of such a kind that it is impossible to obtain the necessary current distribution by a single antenna fed at a single point. The desired result may then often be obtained by using a system of separately fed antennas. For practical reasons a system of identical and identically oriented antennas, a so-called antenna array, is often used for this purpose. The best known type of antenna array is the linear array, i.e., an antenna array in which the antennas are placed along a straight line, and the linear array that has been investigated most thoroughly in the literature is the homogeneous, linear, array; i.e., a linear array with equidistantly placed antennas carrying currents of the same numerical value and with a phase that increases uniformly along the array.

Some antennas consist of identical, but not necessarily identically oriented antennas; such antenna systems will be called quasi-arrays. The present paper deals with an investigation of the field radiated from quasiarrays the elements of which are placed with rotational symmetry, so-called ring quasi-arrays; a special case among them will be a ring array. In particular we shall be interested in ring quasi-arrays the current distribution of which corresponds to the current distribution on a homogeneous, linear array, so-called homogeneous ring quasi-arrays, it being our purpose to ascertain if

$\dagger$ The Technical University of Denmark, Copenhagen, Denmark. such simple ring quasi-arrays have radiated fields of such a type that they can find a practical use as in the case with the corresponding linear arrays. Further we shall investigate the field from a ring quasi-array used as a directional beam-antenna with a principal direction that can be altered by changing the current phases.

We are only interested in considering the field from ring quasi-arrays with so many elements that the field deviates only a little from the field found when the number of elements is infinitely large. Therefore, in each case we shall start by investigating the field for an infinite number of elements. The field existing in the case of a finite number of elements will after that be expressed as the field occurring when the number of elements is infinitely large plus correction terms.

The ring quasi-arrays dealt with here are supposed to be composed of linear antennas. However, most of the characteristic features of these quasi-arrays are found also when the linear antennas are infinitely short; i.e., when the elements of the ring quasi-arrays are Hertz dipoles. For this reason we start by investigating quasi-arrays composed of Hertz dipoles and then proceed to investigate quasi-arrays of linear antennas of finite length.

For homogeneous ring quasi-arrays the field will have its optimum shape when the radius of the quasi-array is infinitely small. Therefore we shall first investigate the field from infinitely small ring quasi-arrays, and next we shall see how the field is deformed when the radius of the quasi-array increases. Although the optimum shape of the radiation diagram is obtained for an infinitely small value of the radius of the quasi-array, it will involve insurmountable, practical difficulties to use a quasi-array with a very small radius. These phenomena will be elucidated on the basis of the theory of super-gain, and by applying this theory we shall find the smallest radius that it is practically possible to use in each case.

As it appears from what has been mentioned above, it is our purpose in the present paper to consider the fields from ring quasi-arrays having certain known current distributions, it being our desire to investigate if quasi-arrays with current distributions of this kind have radiated fields that make such quasi-arrays useful in practice. Hereby we shall meet partly with antenna systems that are already known, which, however, are here brought under a general and well-arranged calculational point of view, partly with antenna systems, that have not been utilized yet, some of which, however, seem to be promising from a practical point of view.

However, instead of this we could have formulated the problem in an opposite way, namely for a given 
ring quasi-array we might wish to find the current distribution that produces a desired radiated field. This problem, with which we shall not deal here, has been made the object of investigation by several authors.

In calculating the radiated field from, and the radiation resistance of, certain ring quasi-arrays we shall find a use for some integrals containing Bessel functions. These integrals are expressed through known and tabulated functions and through the integral of a Bessel function. A table of the integral of the Bessel function of $n$th order has been calculated.

The present paper consists mainly of extracts from the author's treatise on antenna systems with rotational symmetry, ${ }^{1}$ which has been published in Danish. Parts of this treatise have been published in American journals. ${ }^{2-4}$ Problems that have bearing on the investigations described here, have been dealt with in various papers by the author. ${ }^{5-8}$

\section{Ring ARRAY}

\section{Homogeneous Ring Array of Axial Hertz Dipoles}

Radiated Field; The principle of feeding the antennas in a ring array with currents of the same numerical value, but with a phase that increases uniformly along the circle; i.e. as a homogeneous ring array, seems to have been established first by Chireix. ${ }^{9}$ For such a ring array with infinitely many elements Chireix has calculated the radiated field and has thereby shown that this field to an increasing degree will concentrate around the horizontal plane with increasing increment of the current phase per revolution along the circle. Apart from the particular case where the increment of the current phase is zero, the ring array proposed by Chireix is therefore suited as an azimuthally omnidirectional, fadingreducing antenna. An antenna array similar to the one described here, but with several concentric rings, was later proposed independently by Hansen and Woodyard ${ }^{10}$ and investigated further by Hansen and Hollingsworth. ${ }^{11}$ Another type of azimuthally omnidirectional

${ }^{1}$ H. L. Knudsen, "Bidrag til teorien for antennesystemer med hel eller delvis rotationssymmetri," I kommission hos Teknisk Forlag, København; 1953.

2 H. L. Knudsen, "The necessary number of elements in a directional ring aerial," Jour. A ppl. Phys., vol. 22, pp. 1299-1306; 1951.

${ }^{3} \mathrm{H}$. L. Knudsen, "The field radiated by a ring quasi-array of an infinite number of tangential or radial dipoles," PROC. IRE, vol. 41, pp. 781-789; June, 1953 .

${ }^{4} \mathrm{H}$. L. Knudsen, "Radiation resistance of homogeneous ring quasi-array," Proc. IRE, vol. 42, pp. 686-695; April, 1954.

${ }^{5} \mathrm{H}$. L. Knudsen, "The field from a circular and a square helical beam antenna," Trans. Danish Acad. Tech. Sci., no. 8, p. 55; 1950.

"H. L. Knudsen, "Radiation field of a square, helical beam antenna," Jour. Appl. Phys., vol. 23, pp. 483-491; 1952.

${ }^{7} \mathrm{H}$. L. Knudsen, "Superforstaerkning hos antenner," Elektrotek. Tidsskr., vol. 64, pp. 213-221; 1951.

${ }^{8} \mathrm{H}$. 'L. Knudsen, "Shannons tidsopdelingssætning og superforstærkning hos antenner," Tek. Tidsskr., vol. 82, pp. 1023-1030; 1952.

${ }^{\circ}$ H. Chireix, "Antennes a rayonnement zénithal réduit," Onde Elect., vol. 15, pp. 440-456; 1936.

${ }_{10} \mathrm{~W}$. W. Hansen and J. R. Woodyard, "A new principle in directional antenna design," Proc. IRE, vol. 26, pp. 333-345; March, 1938.

${ }^{11} \mathrm{~W}$. W. Hansen and L. M. Hollingsworth, "Design of "flatshooting" antenna arrays," PRoc. IRE, vol, 27, pp. 137-143; February, 1939. antenna array with reduced radiation at high elevation angles may be obtained by feeding a ring array with all currents in the same phase and adding at the center of the circle an antenna carrying a current in phase opposition to the currents in the outer antennas. This antenna array may be considered a special case of one of the arrays investigated by Hansen and Woodyard. The application of this ring antenna as a fading-reducing antenna was described by Böhm ${ }^{12}$ and by Harbich and Hahnemann. ${ }^{13}$

For a homogeneous ring array the discrepancy between the actual array characteristic and the array characteristic which occurs when the number of elements is infinitely large, has already been touched upon by Chireix, ${ }^{9}$ Hansen and Woodyard, ${ }^{10}$ and Hansen and Hollingsworth, ${ }^{11}$ and it has later been investigated in detail by Page. ${ }^{14}$

Carter ${ }^{15}$ has investigated an antenna system composed of a finite number of axial, tangential, or radial dipoles placed around a conducting cylinder, equidistantly along a concentric circle and carrying currents with the same numerical value but with a phase that increases $2 \pi$ during one revolution. In the case where the radius of the conducting cylinder is zero the antenna system with axial dipoles constitutes a particular example of a ring array. In this case the expressions for the radiated field derived by Carter constitute special cases of Page's formulas.

As in the following sections we shall have use for the expression for the array characteristic of a homogeneous ring array with a finite number of elements derived by Page, ${ }^{14}$ and as Page has not used the symbolic method for handling fields with harmonic time variation (the $-i \omega$-method) that is used throughout the following sections of this paper, we shall here give, by using this method, a short derivation of Page's results. The expression for the array characteristic will here be given in a more elaborate form than in Page's work.

The homogeneous ring array considered here is supposed to consist of $s$ identical and identically oriented antennas placed equidistantly along a circle with radius $a$. A spherical co-ordinate system $(r, \theta, \varphi)$ is placed with its origin at the center of the circle, which we shall here and in what follows assume to he horizontal. The coordinate system is supposed to be oriented in such a way that the co-ordinates of the $j$ th antenna are given by $\left(a, \pi / 2, u_{j}\right)$, where

$$
u_{j}=\frac{2 \pi j}{s} \quad j=1,2, \cdots, s,
$$

$12 \mathrm{O}$. Böhm, "Rundfunk-Sendeantennen mit Unterdrückung der Steilstrahlung," Telefunkenztg, vol. 13, pp. 21-26; March, 1932.

${ }^{13} \mathrm{H}$. Harbich and W. Hahnemann, "Wirksame Bekämpfung des Nahschwundes im Rundfunk durch Sendeantennengebilde bestimmter Form," ENT, vol. 9, pp. 361-376; 1932.

${ }^{14} \mathrm{H}$. Page, "Ring-aerial systems. Minimum number of radiators required," Wireless Engr., vol. 25, pp. 308-315; 1948.

${ }_{16}$ P. S. Carter, "Antenna arrays around cylinders," PROC. IRE, vol. 31, pp. 671-693; December, 1943. 
(see Fig. 1). Calling the current in the $j$ th antenna $I_{j}$, according to the definition of a homogeneous array, we can then express $I_{j}$ by

$$
I_{j}=I e^{i H u},
$$

where $I$ is a constant current, and where $H$ is an integer. The angle $u_{j}$ increasing $2 \pi$ when $j$ increases from 0 to $s$, this equation expresses that the current phase increases linearly along the circle and just $H 2 \pi$ during one revolution.

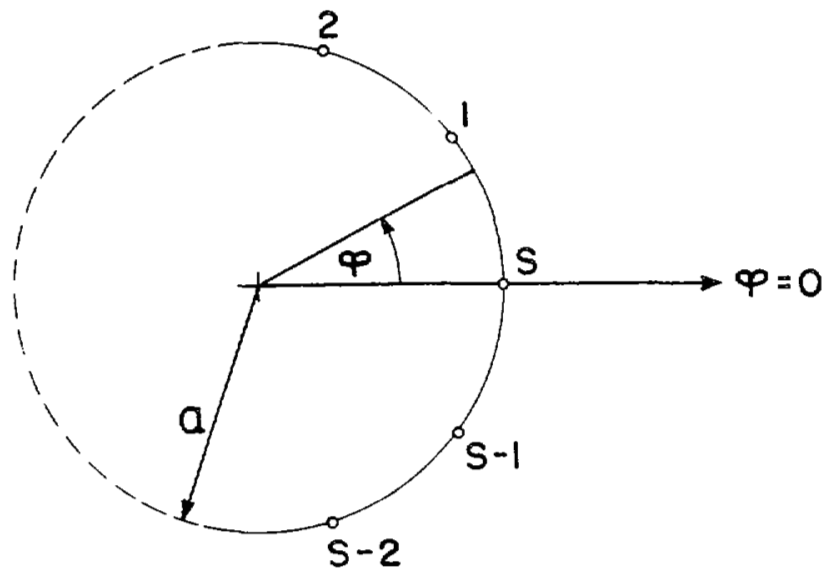

Fig. 1-Ring array with $s$ elements and radius $a$.

Using as a reference antenna an antenna placed at the center of the circle, carrying the current $s I$; i.e., a current with the numerical value equal to the sum of the numerical values of the currents in all of the $s$ elements of the array, we find by using the general expression for the array characteristic of an antenna array the following expression for the array characteristic of the homogeneous ring array in question,

$$
\begin{aligned}
& \mathrm{G}=\sum_{j=1}^{s} \frac{1}{S} e^{i H u_{j}} e^{-i k\left[a \cos u_{j} \sin \theta \cos \phi+o \sin u_{j} \sin \theta \sin \phi\right]} \\
& =\frac{1}{S} \sum_{j=1}^{s} e^{i\left[H u_{j}-z \cos \left(\phi-u_{j}\right)\right]}
\end{aligned}
$$

where for the sake of convenience we have introduced the parameter $z$ thorugh the definition

$$
z=k a \sin \theta .
$$

The parameter $z$ should not be mistaken for the coordinate $z$, which does not occur in the subsequent calculations. By using the following formula known from the theory of Bessel functions,

$$
e^{i z \cos v}=\sum_{n=0}^{\infty}\left(2-\delta_{o n}\right) i^{n} J_{n}(z) \cos n v,
$$

the expression for the array characteristic can be transformed into

$$
\begin{aligned}
G=\sum_{n=0}^{\infty}\left(2-\delta_{o n}\right)(-i)^{n} J_{n}(z) \frac{1}{2 s} \sum_{j=1}^{s} \\
{\left[e^{i\left(n \phi+(H-n) u_{j}\right)}+e^{i\left(-n \phi+(H+n) u_{j}\right)}\right] . }
\end{aligned}
$$

Both of the summations with respect to $j$ are of the type

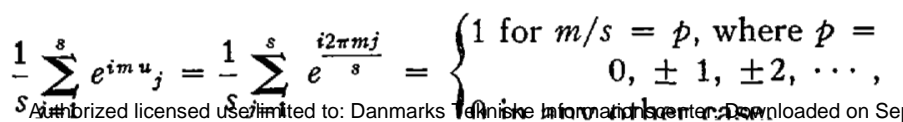

where $m$ denotes an integer. Using this formula and making the assumption

$$
s>|H|
$$

we find after some rearrangement,

$$
\begin{aligned}
G=\frac{1}{2} \sum_{q=0}^{\infty}\left(2-\delta_{o q}\right)\left[J_{H+q g}(z) e^{i(H+q s)(\phi-x / 2)}\right. & \\
& \left.+J_{-H+q s}(z) e^{-i(-H+q s)(\varphi+\pi / 2)}\right] .
\end{aligned}
$$

By multiplying this function with the field from the reference antenna, we obtain the field radiated from the antenna array in question.

Here and in what follows we shall express the electric field strength $\bar{E}(r, \theta, \varphi)$ in the field radiated from an antenna system by

$$
\bar{E}(r, \theta, \varphi)=K \bar{F}(\theta, \varphi) \frac{e^{i k r}}{r},
$$

where $k=\omega \sqrt{\mu \epsilon}$ is the intrinsic propagation constant, $K$ an arbitrarily chosen constant having the dimension of a voltage, and $\bar{F}(\theta, \varphi)$ a vector, being uniquely determined through the choice of $K$, which depends upon the direction $(\theta, \varphi)$, but not upon the distance $r$ to the field point. The component of $\bar{F}$ in the direction of the $r$-axis is zero; consequently, denoting the unit vectors in the directions of the $\theta$ - and the $\varphi$-axes by $\hat{\theta}$ and $\hat{\varphi}$ we can express $\bar{F}$ by $\vec{F}=F_{\theta} \theta+F_{\varphi} \varphi$. We shall denote $\bar{F}$ as the normalized electric field strength and $K$ as the normalization constant. The field will essentially be determined by the specification of $K$ and $\bar{F}(\theta, \varphi)$.

The field from the reference antenna supposed to be a Hertz dipole having the length $L$, carrying the current $s I$, and placed at the center of the ring array in question, is expressed by the normalization constant

$$
K=\frac{i k s L \zeta I}{4 \pi},
$$

where $\zeta=\sqrt{\mu / \epsilon}$ denotes the intrinsic impedance of space, and the normalized, electric field strength $\bar{F}$, where

$$
\begin{aligned}
& F_{\theta}=-\sin \theta, \\
& F_{\varphi}=0 .
\end{aligned}
$$

With the normalization constant chosen here the normalized, electric field strength $\bar{F}$ of the ring array of Hertz dipoles in question will therefore be expressed by

$$
\begin{aligned}
F_{\theta}=-\frac{\sin \theta}{2} \sum_{q=0}^{\infty} & \left(2-\delta_{o q}\right)\left[J_{H+q s}(z) e^{i(H+q s)(\phi-\tau / 2)}\right. \\
& \left.+J_{-H+q s}(z) e^{-i\left(-H^{+q s}\right)(\phi+\pi / 2)}\right], \quad F_{\varphi}=0 .
\end{aligned}
$$

We shall start by considering the field in the case where the number of elements is infinitely large. Accordingly we let $s \rightarrow \infty$ at the same time, letting the length $L$ of the single dipoles converge towards zero in such a way that $s L$ converges towards a finite value. Hereby we find the following limiting values of the components of the normalized, electric field strength

$$
\begin{aligned}
& F_{\theta}=-\sin \theta J_{H}(z) e^{i H(\phi-\pi / 2)}, \\
& F_{\varphi}=0 .
\end{aligned}
$$

This special result was derived already by Chireix. ${ }^{9}$ 
numerical value of the field does not depend on $\varphi$. For $|H| \geqq 1$ points in the horizontal plane having the same phase will be situated on an Archimedes' spiral as is shown in Fig. 2 for respectively $H=1$ (full line) and $H=-1$ (dotted line). Sandeman ${ }^{16}$ and Granquist ${ }^{17}$ have suggested utilizing this fact for navigational purposes.

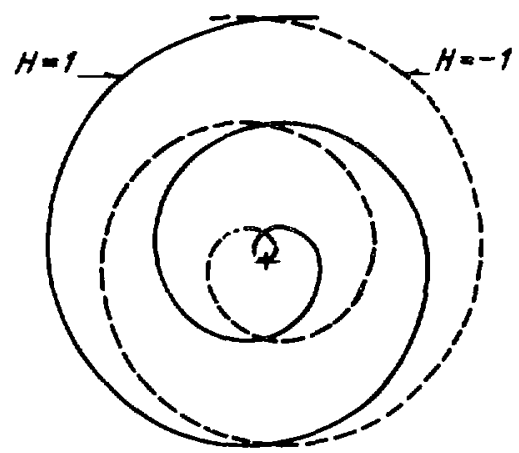

Fig. 2-Equi-phase lines for a homogeneous ring quasi-array with $\mathrm{H}= \pm 1$.

If the radius of the ring array is much smaller than the wavelength $\lambda$ divided by $2 \pi$; i.e., if $k a \ll 1$, the Bessel function in the expression given above can be expressed with sufficient accuracy by the first term of its series

$$
\begin{aligned}
& F_{\theta} \cong-\frac{(k a)^{H}}{2^{H} H !} \sin ^{H+1} \theta e^{i H(\varphi-\pi / 2)}, \\
& F_{\varphi}=0 .
\end{aligned}
$$

In Fig. 3(a)-3(d) the numerical value of $F_{\theta}$ is plotted as a function of $\theta$ for various values of $H$. The figure shows that the field, which everywhere is polarized in the $\theta$-direction, to an increasing degree will concentrate around the horizontal plane when the increment of the current phase per revolution, as expressed by $H$, increases. As pointed out by Chireix, for $|H| \geqq 1$ the ring array described here is therefore suited as a fadingreducing antenna for broadcasting purposes. The homogeneous ring array with $H=0$; i.e., with all the currents in phase, is not suited as a fading-reducing antenna, this array having the same polar diagram as a single dipole. However, as was mentioned above, when combining it with a center antenna, it is possible to obtain an antenna array having zero radiation at a given elevation angle and consequently reduced radiation at high elevation angles. Large antenna systems of this type are at present being planned by the Swedish Telegraph Administration and by Nordwestdeutscher Rundfunk in Western Germany.

If the radius of the ring array is not infinitely small, as was assumed above, the field will not assume the ideal shape shown in Fig. 3, but deviate from it to an increasing degree as the radius increases. However, if we choose as small a radius as the consideration of avoiding super-gain permits, the polar diagrams will deviate only to a small degree from those shown in Fig. 3.

${ }^{16}$ E. K. Sandeman, "Spiral-phase fields," Wireless Engr., vol, 26, pp. $96-105 ; 1949$.

${ }_{17}$ C. E Granquist, "Radiofyr för avstånd och riktning" Paper

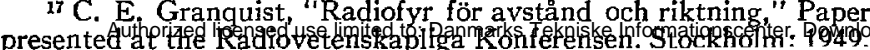

So far we have assumed that the number of elements is infinitely large. However, in practice the number of elements will be finite; this implies that the antenna system will no longer be exactly azimuthally omnidirectional as in the case of infinitely many elements. Economical considerations usually make it desirable to reduce the number of elements as much as possible. It is therefore of interest to investigate the influence of the number of elements on the shape of the radiated field. In order that the field from the homogeneous ring array in question with a finite number of elements may be a fair approximation to the field from the corresponding array with infinitely many elements, the term corresponding to $q=0$, which, as shown above, is identical with the field in the case of $s=\infty$, must be the dominant term in the infinite series. The approximate condition that this will occur is that

$$
s>2|H|
$$

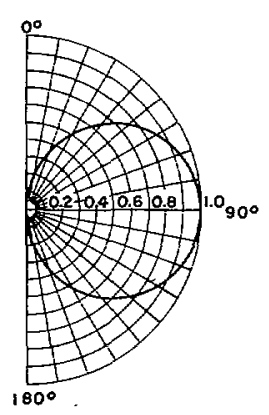

a. $H=0$.

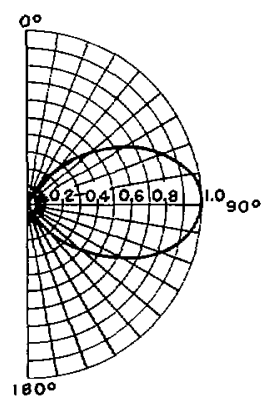

c. $H=2$.

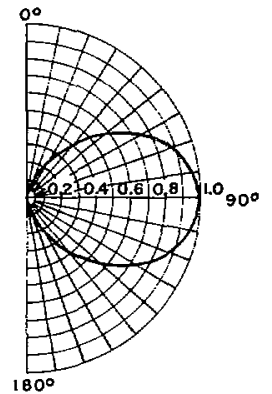

b. $H=1$.

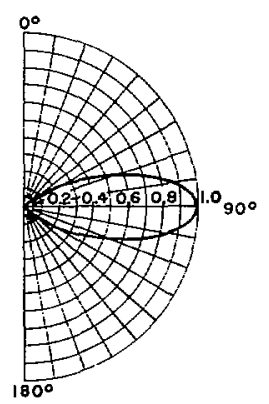

d. $H=9$.
Fig. 3-The field radiated from a small, homogeneous ring array of axial dipoles for various values of $H$.

the Bessel function occurring in the term corresponding to $q=0$, then, is of a smaller order than any of the Bessel functions appearing in the correction terms. In what follows we shall assume that this inequality is satisfied, and not only the less strong inequality introduced above, $s>|H|$. Subsequently we shall consider only such cases where the correction terms are small as compared with the principal term; apart from the main term it will then be necessary in general to include only the first correction term. The field is then approximately expressed by

$F_{\theta} \cong-\sin \theta e^{i H \phi}\left\{(-i)^{B} J_{H}(z)+(-i)^{s}\left[(-i)^{H} e^{i s \phi} J_{H+s}(z)\right.\right.$ 
Whereas the numerical value of the array characteristic in the case of $s=\infty$ is independent of $\varphi$, as was mentioned above, the characteristic for the array considered here will oscillate weakly with $\varphi$ for any fixed value of $\theta$.

For a function $f(\varphi)$ that oscillates weakly between two boundaries $f_{\min }$ and $f_{\max }$; i.e.,

where

$$
f_{\min } \leqq f(\varphi) \leqq f_{\max }
$$

$$
2 \delta f=f_{\max }-f_{\min } \ll f(\varphi),
$$

we shall introduce for use here and in the following sections a parameter, the so-called relative, maximum variation $\Delta f$, to describe the relative change of the function

$\Delta f=\frac{f_{\mathrm{max}}-f_{\mathrm{min}}}{\left(f_{\mathrm{max}}+f_{\min }\right)} \cong \frac{f_{\mathrm{max}}-f_{\mathrm{min}}}{2 f_{\mathrm{max}}} \cong \frac{f_{\mathrm{max}}-f_{\mathrm{min}}}{2 f_{\min }}$.

In calculating the relative, maximum variation $\Delta\left|F_{\theta}\right|$ of the field radiated from the ring array in question we must consider the cases $H=0$ and $|H| \geqq 1$ separately.

$H=0$. The approximate expression for the field derived above may in the present case be written as follows

$$
F_{\theta} \cong-\sin \theta\left[J_{0}(z)+(-i)^{s} 2 J_{\mathrm{s}}(z) \cos s \varphi\right] .
$$

Here we must treat the cases of $s$ even and $s$ odd separately.

$s$ even. In the case of $s$ even the field may be expressed with advantage by

$$
F_{\theta} \cong-\sin \theta\left[J_{o}(z)+(-1)^{s / 2} 2 J_{s}(z) \cos s \varphi\right] .
$$

This expression shows that the correction term is in phase with or in phase opposition to the principal term for any value of $\varphi$. From this we find for the relative, maximum variation $\Delta\left|F_{\theta}\right|$,

$$
\Delta\left|F_{\theta}\right| \cong \frac{2\left|J_{s}(z)\right|}{\left|J_{o}(z)\right|}
$$

$s$ odd. When $s$ is odd, it is convenient to transform the expression for the field as follows,

$$
F_{\theta}=-\sin \theta\left[J_{o}(z)+i(-1)^{s+1 / 2} 2 J_{s}(z) \cos s \varphi\right] .
$$

This expression shows that in the present case the correction term is in phase quadrature with the principal term for any value of $\varphi$. Therefore in the present case the relative, maximum variation $\Delta\left|F_{\theta}\right|$, is expressed by

$$
\Delta\left|F_{\theta}\right| \cong \frac{\left(J_{s}(z)\right)^{2}}{\left(J_{o}(z)\right)^{2}} .
$$

By comparing the expressions for $\Delta\left|F_{\theta}\right|$ derived here for the cases of $s$ even and $s$ odd we see that for the same ratio of the numerical value of the correction term to the numerical value of the principal term, the relative, maximum variation of the field is essentially smaller in the case of $s$ odd than in the case of $s$ even. This fact appears also from Fig. 4, where the relative, maximum variation for a homogeneous ring array with $H=0$; i.e., with currents in the same phase, is plotted as a function of $a / \lambda \sin \theta$. The curves show that three antennas produce a less wavy field than four antennas, five antennas a better field than six, and seven antennas a better field than eight. This peculiar and practically important fact, which has not always been taken into account, was pointed out by Page. ${ }^{14}$

$|H| \geqq 1$. In the case, $H=0$, considered above the numerical values of the two correction terms containing $J_{H+s}(z)$ and $J_{-H+s}(z)$ were of the same magnitude, for which reason both of them had to be taken into account.

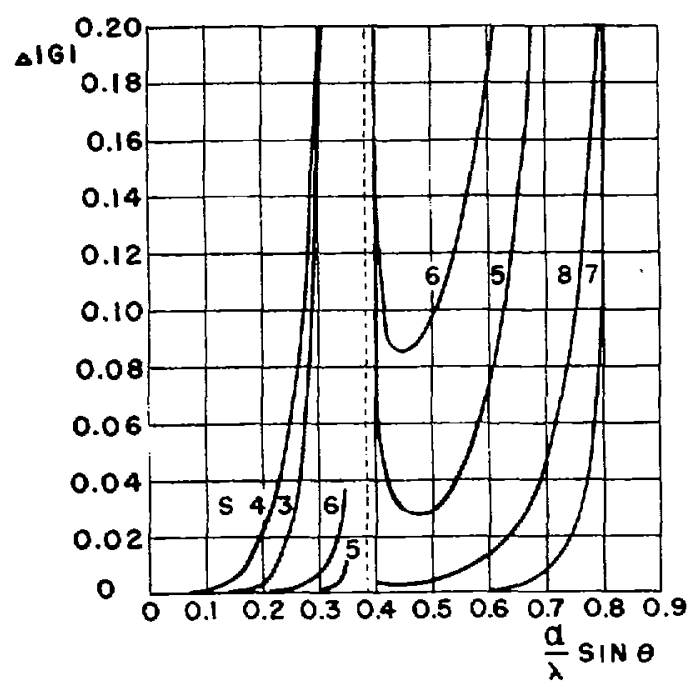

Fig. 4-The relative, maximum variation $\Delta\left|F_{\theta}\right|$ for a homogeneous ring array for $H=0$.

However, for $H \geqq 1$ it will generally hold good that the correction term containing the function $J_{-H+s}(z)$ will be much larger than the term containing $J_{H+s}(z)$, the numerical value of a Bessel function with a constant argument decreasing fast though not necessarily monotonically with increasing order. Correspondingly, for $H \leqq-1$ it will generally hold good that $J_{H+s}(z)$ will be much larger than $J_{-H+s}(z)$. Thus we find the following approximate expressions for the normalized electric field strength ;

$F_{\theta} \leqq-\sin \theta\left[(-i)^{B} e^{i H_{\varphi}} J_{H}(z)\right.$
$+(-i)^{\mp} z^{+s} e^{\left.\mp i\left(\mp H^{+s}\right)^{\varphi} J_{\mp H+8}(z)\right]}$
$=\left\{\begin{array}{r}-\sin \theta(-i)^{H} e^{i H \varphi}\left[J_{H}(z)+(-1)^{H}(-i)^{s} e^{-i s \varphi} J_{-H+s}(z)\right] \\ \text { for } H=1, \\ -\sin \theta(-i)^{H} e^{i H \varphi}\left[J_{H}(z)+(-i)^{s} e^{i s \varphi} J_{H+s}(z)\right] \\ \text { for } H=-1 .\end{array}\right.$

From the expression for the field derived here we see that the numerical value of the correction term is constant, whereas the phase difference between the principal term and the correction term will increase linearly with $\varphi$. The ploar diagram of the numerical value of the field is an undulating line in the shape of a circle with one period between two neighboring antennas. The extreme values will occur in the directions that are $\pi / 2$ behind the directions towards the antennas and in the directions halfway between these directions. The relative, maximum variation is found to be

$$
\left|F_{\theta}\right|=\frac{\left|J_{\mp H+s}(z)\right|}{\left|J_{H}(z)\right|} .
$$


This expression being valid for an even as well as for an odd number of antennas, it is seen that in the present case no systematic advantage can be derived from using an odd instead of an even number of elements as was the case for $H=0$. The relative, maximum variation in the case of $H=4$ is plotted in Fig. 5 .

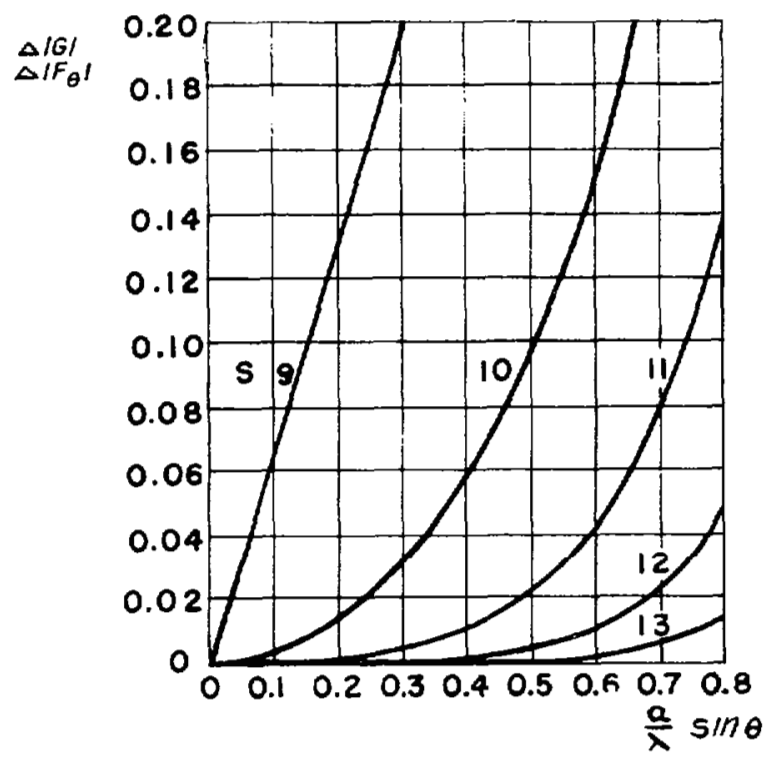

Fig. 5-The relative, maximum variation $\Delta\left|F_{\theta}\right|$ for a homogeneous ring array for $|H|=4$.

Radiation resistance. For a homogeneous ring array of infinitely many Hertz dipoles Page has carried out a calculation of the radiation resistance. ${ }^{18}$ From the derived expression it appears that the radiation resistance increases with increasing radius $a$ of the ring array, and that it decreases with increasing increment of the current phase per revolution; i.e., with increasing $H$.

\section{Directional Ring Array}

Stenzel has shown that a ring array may be utilized for obtaining an antenna system with pronounced directional properties. ${ }^{19}$ By giving the currents of the antennas in the ring a phase distribution such that there is constructive interference of the waves emitted in an arbitrary direction in space, hereafter called the principal direction, an array characteristic is obtained having a principal lobe in this direction. Stenzel considers especially the case where the principal direction is horizontal. If the principal direction is rotated in the horizontal plane, the horizontal diagram of the array characteristic will also rotate and, as a consequence of the approximate rotational symmetry of the ring aerial, with its shape almost unchanged. For the ring aerial mentioned here, Stenzel has investigated the discrepancy between the array characteristic in the case of a finite, even number of elements and the characteristic in the case of infinitely many elements, apparently assuming that there is no essential difference between the case of an even number of elements and that of an odd number of elements. This assumption is explicitly stated by Brückmann in his textbook on antennas in the chapter dealing with Stenzel's theory. ${ }^{20}$ In the present paper it will be investigated whether the array characteristic of the directional ring aerial with an even number of antennas differs essentially from the characteristic of the ring aerial with an odd number of elements as was the case with the ring aerial investigated by Page. ${ }^{14}$ This investigation is based on Stenzel's as well as on Page's theory.

Let $s$ identical and identically oriented antennas be placed along a circle with a radius $a$ as shown in Fig. 1, and let us introduce a spherical co-ordinate system as described above in connection with this figure. The current $I_{j}$ in the $j$ th antenna is expressed by

$$
I_{j}=I e^{i \delta j}
$$

where $I$ is a constant, whereas $\delta_{j}$ denotes the current phase of the $j$ th antenna. Choosing as a reference antenna, an antenna placed at the center of the circle and carrying a current $s I$ we find by using the conventional expression for the array characteristic $G(\theta, \varphi)$ of an antenna array the following expression for the array characteristic of the ring array considered here;

$$
G=\frac{1}{s} \sum_{j=1}^{s} e^{i\left(\delta_{j}-k a \sin \theta \cos \left(\phi-u_{j}\right) l\right.},
$$

where, as above, $k=\omega \sqrt{\mu \epsilon}$ denotes the intrinsic propagation constant of space.

In accordance with Stenzel's suggestion, ${ }^{19}$ the currents in the $s$ antennas are now given such phases that the waves emitted in an arbitrarily chosen direction $\left(\theta_{o}, \varphi_{o}\right)$, the principal direction, are in phase. This is obtained by choosing

$$
\delta_{j}=k a \sin \theta_{o} \cos \left(\varphi_{o}-u_{j}\right)
$$

By this choice of the phases $\delta_{j}$ the expression for the array characteristics will be

$$
G=\frac{1}{s} \sum_{j=1}^{s} e^{i k a\left[\sin \theta_{o} \cos \left(\phi_{o}-u_{j}\right)-\sin \theta \cos \left(\phi-u_{j}\right)\right]}
$$

Following Stenzel in defining an angle $\xi$ by the equation

$$
\cos \xi=\frac{\sin \theta \cos \varphi-\sin \theta_{0} \cos \varphi_{0}}{\sqrt{\left(\sin \theta \cos \varphi-\sin \theta_{0} \cos \varphi_{0}\right)^{2}+\left(\sin \theta \sin \varphi-\sin \theta_{0} \sin \varphi_{0}\right)^{2}}},
$$

and a parameter $\rho$ by the equation

$$
\rho=a \sqrt{\left(\sin \theta \cos \varphi-\sin \theta_{o} \cos \varphi_{o}\right)^{2}+\left(\sin \theta \sin \varphi-\sin \theta_{o} \sin \varphi_{o}\right)^{2}}
$$

\footnotetext{
${ }^{18} \mathrm{H}$. Page, "Radiation resistance of ring aerials," Wireless Engr, vol. 25, pp. 102-109; 1948.

${ }_{19} \mathrm{H}$. Stenzel, "Uber die Richtkarakteristik von in einer Ebene angeordneten Strahlern," ENT,vol. 6, pp. 165-181; 1929.
}

${ }^{20} \mathrm{H}$. Brückmann, "Antennen. Ihre Theorie und Technik, Leipzig, p. 113; 1939. 
we may now express the array characteristic in the following way,

$$
G=\frac{1}{s} \sum_{j=1}^{s} e^{-i k_{\rho} \cos \left(\xi-u_{j}\right)}
$$

This expression is of a type similar to the expression derived above for the array characteristic for a homogeneous ring array with antenna currents having the same phase; i.e., with $H=0$. Using a similar procedure to that for the homogeneous ring array, we may write the expression derived here

$$
G=\sum_{q=0}^{\infty}\left(2-\delta_{o n}\right)(-i)^{g s} J_{q s}(k \rho) \cos q s \xi .
$$

If the number $s$ of elements is infinitely large, the array characteristic will be expressed by the first term in this infinite series

$$
G=J_{o}\left(k_{\rho}\right) \text {. }
$$

When the number of elements is finite, the remaining terms in the series will express the deviation of the array characteristic from the array characteristic applying in the case of an infinite number of elements.

The array characteristic in the case of a finite number of elements may be transformed with advantage so that it clearly appears which terms are in phase with (or in phase opposition to), and which are in phase quadrature with, the principal term. In so doing we must distinguish between the cases where the number $s$ of elements is even and those where it is odd.

s even.

$G=J_{o}(k \rho)+2 \sum_{q=1}^{\infty} J_{q s}\left(k_{\rho}\right) \cos \left(\frac{\pi}{2}-\xi\right) q s$.

s odd.

$$
\begin{array}{r}
G=J_{o}(k \rho)+2 \sum_{q=1}^{\infty} J_{2 q s}(k \rho) \cos \left(\frac{\pi}{2}-\xi\right) 2 q s \\
-i 2 \sum_{q=0}^{\infty} J_{(2 q+1)}(k \rho) \sin \left(\frac{\pi}{2}-\xi\right)(2 q+1) s .
\end{array}
$$

The expression for the array characteristic for $s$ even was derived by Stenzel approximately in the way described here. The correction terms in the case of $s$ even are seen to be in phase with the principal term. On the other hand, in the case of $s$ odd, there occur correction terms in phase with, as well as correction terms in phase quadrature with, the principal term. Let us for a moment suppose that we have chosen $s$ so large that the correction terms are small compared with the maximum value of the principal term. The array characteristic may then be expressed sufficiently accurately by the principal term and the first term in each of the infinite series of correction terms.

s even.

$$
G \cong J_{o}\left(k_{\rho}\right)+2 J_{s}\left(k_{\rho}\right) \cos \left(\frac{\pi}{2}-\xi\right) s
$$

s odd.

$$
G \cong J_{o}(k \rho)-i 2 J_{s}(k \rho) \sin \left(\frac{\pi}{2}-\xi\right) s
$$

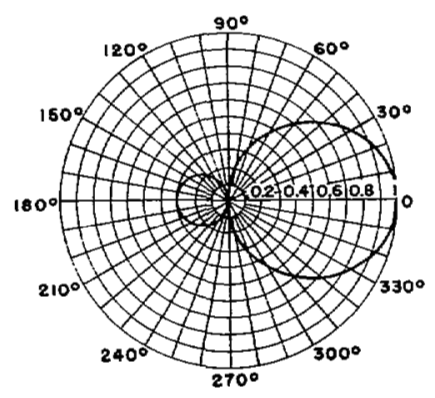

a. $\frac{a}{\lambda}=0.25$.

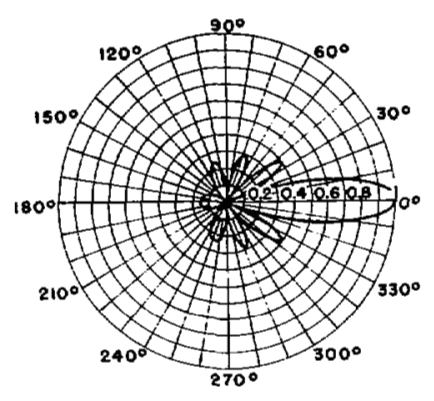

b. $\frac{a}{\lambda}=1$.

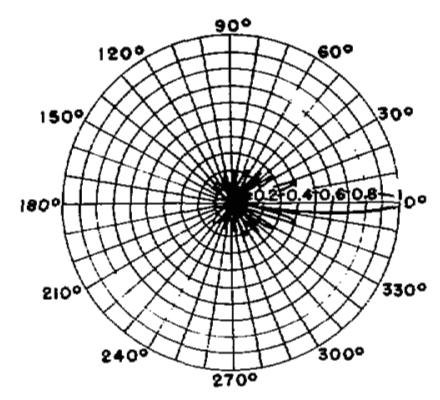

c. $\frac{a}{2}=2$.
Fig. 6-Horizontal diagram of the array characteristic for a directional ring array for various values of $a / \bar{\lambda}$.

As the most important correction term in the case of $s$ even is in phase with the principal term, whereas the most important correction term in the case of $s$ odd is in phase quadrature with the principal term, the latter case must be assumed to be more favourable than the former in regard to the approximation of the array characteristic to the array characteristic valid for $s=\infty$. This will be illustrated later by a numerical example.

When the principal direction is vertical; i.e., when $\theta_{o}=0$, the array will constitute a homogeneous ring array with currents in the same phase. The expression for the array characteristic derived here will then be reduced to the expression derived above for the array characteristic for a homogeneous ring array with $H=0$. In what follows we shall illustrate the expression for the array characteristic by investigating a ring array with its principal direction in the horizontal plane.

For a ring array with a horizontal principal direction; i.e., with $\theta_{0}=\pi / 2$, we find, confining the investigation to the field in the horizontal plane $\theta=\pi / 2$,

$$
\begin{aligned}
& \xi=\frac{\pi}{2}+\frac{\varphi+\varphi_{0}}{2}, \\
& \rho=2 a \sin \frac{\varphi-\varphi_{0}}{2} .
\end{aligned}
$$

For a ring array with infinitely many elements and with the quotient between the radius and the wavelenth $a / \lambda=0.25,1$, and 2 we hereby find the horizontal patterns of the array characteristic shown in Fig. $6(a)-6(c)$. By rotating the distribution of current phases 
these horizontal patterns will make a corresponding rotation in the horizontal plane. If the number of elements is infinitely large, this rotation will take place without any deformation of the horizontal pattern of the array characteristic. On the other hand, if the number of elements is finite, the radiation pattern will vary periodically, when the distribution of current phases is rotated.

On the basis of the exact expression for the array characteristic, the array characteristic has been calculated for a ring array with $a / \lambda=0.5$ and with respectively $5,6,7$, and 8 elements for various values of the azimuth $\varphi_{0}$ of the principal direction. The horizontal patterns found in this way are plotted in Fig. 7(a)-7(h). In this figure the principal direction is fixed, whereas the antenna system rotates. The figure shows that for a small number of antennas, $s=5$ or 6 , the radiation pattern deviates considerably from the pattern valid for $s=\infty$. However, it is seen that 5 antennas give almost as good an approximation to the ideal pattern as 6 , and 7 antennas almost as good an approximation as 8 . This confirms the assumption that an odd number of antennas is preferable to an even number. The advantage of using an odd number is, however, not so great here as in the case of the homogeneous ring array dealt with above.

\section{Homogeneous Ring Quasi-ArRay of Tangential Antennas}

\section{Homogeneous Ring Quasi-Array of Tangential Hertz Dipoles}

Radiated Field; In a preceding section we have seen that by using a homogeneous ring array of axial Hertz dipoles we can obtain an azimuthally omnidirectional, horizontally polarized field that is concentrated around the horizontal plane. During recent years FM and television have aroused an interest in antennas that radiate horizontally polarized fields. It is therefore an obvious thing in analogy to the investigation made above of the field from a ring array of axial dipoles to investigate the field from a ring quasi-array of equidistant, tangential Hertz dipoles carrying currents of the same numerical value and with a phase that increases uniformly along the circle. A ring array of this kind will be called a homogeneous ring quasi-array. In analogy with what was done in the case of the homogeneous ring array the increment of the current phase during one revolution will be denoted by $H 2 \pi$, where $H$ is an integer.

As mentioned above, Carter has carried through a calculation of the field from a ring array of a finite number of axial dipoles and from a ring quasi-array of a finite number of tangential or radial dipoles placed concentrically around a conducting cylinder and with $H=0$ or $H=1 .{ }^{15}$ In the case where the radius of the conducting cylinder is equal to zero, and where the current phases are characterized by $H=0$ or $H=1$, the antenna systems treated by Carter constitute special cases of the systems investigated here.

The field from an arbitrarily large, circular frame

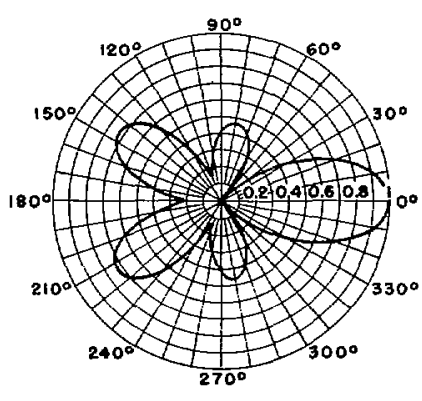

a. $s=5$,
$\varphi_{0}=0^{\circ}$

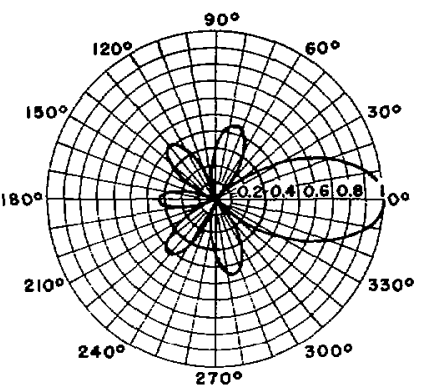

c. $s=6$,

$\varphi_{0}=0^{\circ}$.

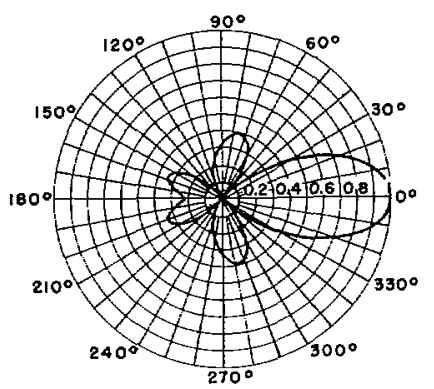

e. $\begin{aligned} & s=7 \\ & \varphi_{0}\end{aligned}=\dot{0}^{\circ}$

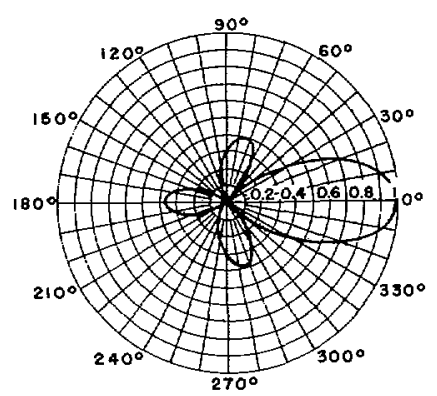

b. $\begin{aligned} s & =B \\ \varphi_{0} & =0^{\circ} .\end{aligned}$

Fig. 7-Horizontal diagram of the array characteristic for a directional ring array with $a / \lambda=0.5$ for various values of the number $s$ of elements.

aerial with constant current (i.e., $H=0$ ) has previously been investigated by Foster ${ }^{21}$ and Moullin. ${ }^{22}$ This field calculation is included in this paper as a special case.

\footnotetext{
${ }^{21}$ D. Foster, "Loop antennas with uniform current," Proc. IRE, vol. 32 pp. 603-607; October, 1944.

${ }^{22}$ E. B. Moullin, "Radiation from large circular loops" Jour. I. E. E., Pt. III, vol.93, pp. 345-351; 1946.
} 
The homogeneous ring quasi-array considered here is assumed to consist of $s$ identical and identically oriented Hertz dipoles having the length $L$ and being placed equidistantly along a circle with radius $a$ tangential to this circle, as shown in Fig. 8. A spherical

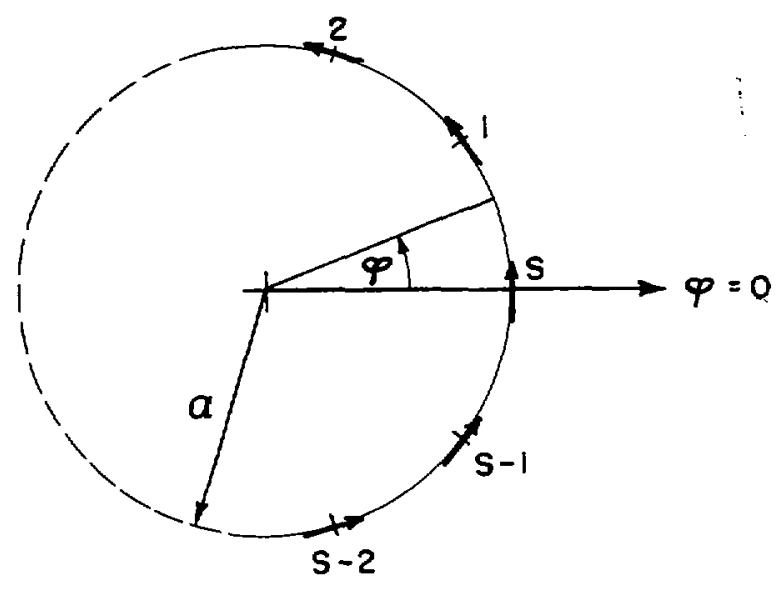

Fig. 8-Ring quasi-array of $s$ tangential dipoles.

co-ordinate system $(r, \theta, \varphi)$ is placed as shown in the figure in the same way as the co-ordinate system shown in Fig. 1 so that the co-ordinates of antenna $j$ are given by $\left(a, \pi / 2, u_{j}\right)$, where

$$
u_{j}=\frac{2 \pi j}{s} \quad j=1,2, \cdots, s
$$

It is assumed that the current $I_{j}$ in the $j$ th dipole is given by

$$
I_{j}=I e^{i H u_{j}},
$$

where $I$ is a constant and $H$ an integer, as mentioned above. We express the electric field strength $\bar{E}$ at the point $(r, \theta, \varphi)$ by using the notation introduced above,

$$
\bar{E}(r, \theta, \varphi)=K \bar{F}(\theta, \varphi) \frac{e^{i k r}}{r},
$$

where $K$ is a normalization constant and $\vec{F}$ the dimension-free, normalized electric field strength. As in the investigation of the homogeneous ring array we choose here the normalization constant

$$
K=\frac{i k s L \zeta I}{4 \pi}
$$

In a rectangular co-ordinate system placed in the usual way in relation to the spherical co-ordinate system we find by conventional methods the following expression for the components of the normalized electric field strength;

$F_{x}=-\sum_{j=1}^{s} \frac{1}{s} e^{i\left[H u_{j}-k a\left(\cos u_{j} \sin \theta \cos \varphi+\sin u_{j} \sin \theta \sin \varphi\right)\right]} \sin u_{j}$,

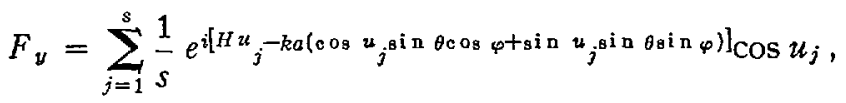
$F_{z}=0$,

or, by using the short-hand notation used above

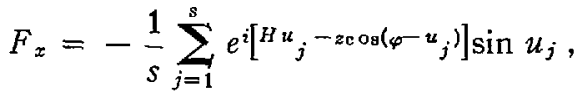

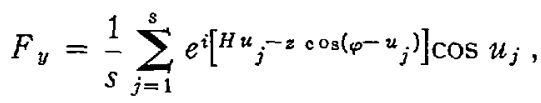

$$
\begin{aligned}
& F_{z}=0 \text {. }
\end{aligned}
$$

By using the series development given above,

$$
e^{i \varepsilon \cos v}=\sum_{n=0}^{\infty}\left(2-\delta_{o n}\right) i^{n} J_{n}(z) \cos n v,
$$

we may express the rectangular components of $\bar{F}$ by

$$
\begin{aligned}
& F_{x}=-\frac{1}{s} \sum_{j=1}^{s} e^{i H u_{j}} \sin u_{j} \sum_{n=0}^{\infty}\left(2-\delta_{0 n}\right)(-i)^{n} J_{n}(z) \\
& \cos n\left(\varphi-u_{j}\right) \\
& =\frac{1}{4 s} \sum_{n=0}^{\infty}\left(2-\delta_{o n}\right)(-i)^{n+1} J_{n}(z) \sum_{j=1}^{s}\left\{e ^ { i n _ { \varphi } } \left[-e^{i(H-n+1) u_{j}}\right.\right. \\
& \left.\left.+e^{i(H-n-1) u_{j}}\right]+e^{-i n_{\varphi}[}\left[-e^{i(H+n+1) u_{j}}+e^{i(H+n-1) u_{j}}\right]\right\} \text {, } \\
& F_{y}=\frac{1}{s} \sum_{j=1}^{s} e^{i H u_{j}} \cos u_{j} \sum_{n=0}^{\infty}\left(2-\delta_{o n}\right)(-i)^{n} J_{n}(z) \\
& =\frac{1}{4 s} \sum_{n=0}^{\infty}\left(2-\delta_{o n}\right)(-i)^{n} J_{n}(z) \\
& \sum_{j=1}^{s}\left\{e^{i n_{\varphi}}\left[e^{i(H-n+1) u_{j}}+e^{i(H-n-1) u_{j}}\right]\right. \\
& \left.+e^{-i n_{\varphi}}\left[e^{i(H+n+1) u_{j}}+e^{i(H+n-1) u_{j}}\right]\right\} \text {, } \\
& F_{z}=0 \text {. } \\
& \cos n\left(\varphi-u_{j}\right)
\end{aligned}
$$

The summations with respect to $j$ in the above expressions are all of the type previously described;

$$
\begin{array}{r}
\frac{1}{s} \sum_{j=1}^{s} e^{i m u_{j}=\frac{1}{s} \sum_{j=1}^{s} \frac{e^{i 2 \pi m j}}{s}} \\
=\left\{\begin{array}{l}
1 \text { for } m / s=p, \text { where } \\
p=0, \pm 1, \pm 2, \cdots, \\
0 \text { in any other case, }
\end{array}\right.
\end{array}
$$

where $m$ denotes an integer. By using this formula together with the following recursion formulas for Bessel functions,

$$
\begin{gathered}
J_{n-1}(x)+J_{n+1}(x)=\frac{2 n}{x} J_{n}(x), \\
J_{n-1}(x)-J_{n+1}(x)=2 J_{n}^{\prime}(x),
\end{gathered}
$$

and by assuming that

$$
s>|H|+1 \text {, }
$$

we find, after some manipulation,

$$
\begin{gathered}
F_{x}=\frac{1}{2} \sum_{q=0}^{\infty}\left(2-\delta_{o q}\right)\left\{\left[\frac{H+q s}{z} J_{H+q s}(z) \cos \varphi\right.\right. \\
\left.\quad-i J_{H+q s}^{\prime}(z) \sin \varphi\right] \\
e^{i(H+q s)(\varphi-\pi / 2)}+\left[-\frac{-H+q s}{z} J_{-H+q s}(z) \cos \varphi\right. \\
\left.\left.-i J^{\prime}{ }_{-H+q s}(z) \sin \varphi\right] e^{-i(-H+q s)(\varphi+\pi / 2)}\right\}, \\
F_{y}=\frac{1}{2} \sum_{q=0}^{\infty}\left(2-\delta_{o q}\right)\left\{\left[i J_{H+q s}^{\prime}(z) \cos \varphi\right.\right.
\end{gathered}
$$




$$
\begin{gathered}
\left.+\frac{H+q s}{z} J_{H+q s}(z) \sin \varphi\right] \\
e^{i(H+q s)(\varphi-\pi / 2)}+\left[i J^{\prime}-H+q s(z) \cos \varphi\right. \\
\left.-\frac{-H+q s}{z} J_{-H+q s}(z) \sin \varphi\right] \\
\left.e^{-i(-H+q s)(\varphi+\pi / 2)}\right\}, \\
F_{z}=0 .
\end{gathered}
$$

From the expressions for the rectangular components of the normalized electric field strength obtained here we find the following expressions for its spherical components:

$$
\begin{gathered}
F_{A}=F_{x} \cos \theta \cos \varphi+F_{y} \cos \theta \sin \varphi-F_{z} \sin \theta \\
=\frac{1}{2} \cos \theta \sum_{q=0}^{\infty}\left(2-\delta_{o q}\right)\left[\frac{H+q s}{z} J_{H+q s}(z) e^{i(H+q s)(\phi-\pi / 2)}\right. \\
\left.-\frac{-H+q s}{z} J_{-H+q s}(z) e^{-i(-H+q s)(\phi+\pi / 2)}\right] \\
=\frac{i}{2} \sum_{q=a}^{\infty}\left(2-\delta_{o q}\right)\left[J_{H+q s}^{\prime}(z) e^{i(H+q s)(\phi-\pi / 2)}\right. \\
\left.\quad+J_{-H+q s}^{\prime}(z) e^{-i(-H+q s)(\phi+\pi / 2)}\right] .
\end{gathered}
$$

Let us first consider the case where the number of elements is infinitely large. Any other term in the above expressions except those corresponding to $q=o$ will then be zero, and we find

$$
\begin{gathered}
F_{\theta}=\frac{H}{z} J_{H}(z) \cos \theta e^{i H(\phi-\pi / 2)}, \\
F_{\varphi}=i J^{\prime}{ }_{H}(z) e^{i_{H}\left(^{(\phi-\pi / 2)} .\right.}
\end{gathered}
$$

These expressions show that in general both the $\theta$ - and the $\varphi$-components of the electric field strength will be different from zero. Further, as they are in phase quadrature with each other, the radiated field will in general be elliptically polarized. Only in the case of $H=0$; i.e., for a circular frame aerial with a constant current, the $\theta$-component of the electric field strength is zero in any direction, so that the field will be horizontally polarized everywhere. For $|H| \geqq 1$, in the horizontal plane, points having the same phase will be situated on an Archimedes' spiral as shown in Fig. 2 for the case $H= \pm 1$. The numerical values of the field components will be independent of $\varphi$.

In particular we shall consider the case, where the radius $a$ of the ring quasi-array is much smaller than the wavelength $\lambda$ divided by $2 \pi$; i.e., where $k a \ll 1$. The fact is that it is appropriate to let the radius of the ring quasi-array have so small a value that the polar diagram of its radiated field deviates little from the polar diagram occurring in the case of an infinitely small radius. The Bessel functions in the expressions derived above may then approximately be replaced by the first term of their series development, so that we find

$$
F_{\theta}= \begin{cases}0 & \text { for } H=0, \\ \frac{(k a)^{H-1}}{2^{H}(H-1) !} \cos \theta \sin ^{H-1} \theta e^{i H(\phi-\pi / 2)} & \text { for }|H| \geqq 1,\end{cases}
$$

$F_{\varphi}= \begin{cases}-i \frac{k a}{2} \sin \theta & \text { for } H=0, \\ i \frac{(k a) H^{-1}}{2 H(H-1) !} \sin ^{H^{-1}} \theta e^{i H(\phi-\pi / 2)} & \text { for }|H| \geqq 1 .\end{cases}$

The numerical values of $F_{\theta}$ and $F_{\varphi}$ are plotted in Fig. 9(a) and $(c)-(e)$ as functions of $\theta$ for various values of $H$. For any value of $H$ the field will be horizontally polarized in horizontal directions. For $H=0$ we obtain the wellknown polar diagram of the radiated field from a small frame aerial. In the case where $|H|=1$, and in this case only, the radiation in the direction of the axis is different from zero; in this case the field will be circularly

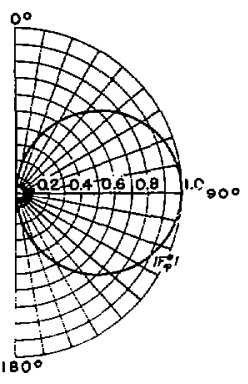

a. TANG. DIPOLES, $H=O$

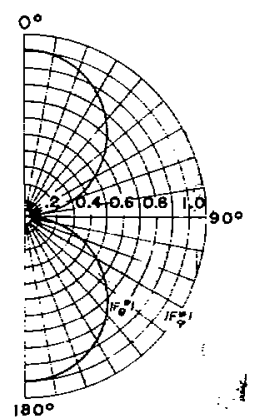

C. TANG.AHDRAD. DIPOLES, $H=\mathbf{I}$.

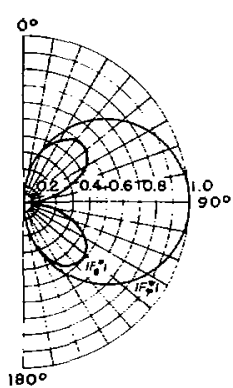

d. TANG. ANO RAD. DIPOLES, $\mathrm{H}=2$.

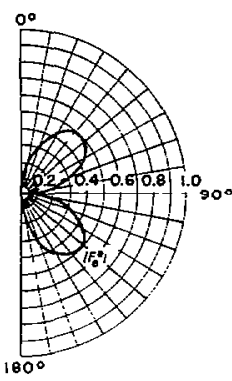

b. RAD. DIPOLES, $H=O$.

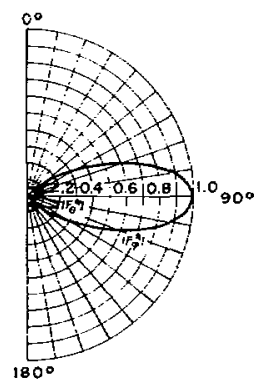

E.TANG. AND RAD. DIPOLES, $H=9$.
Fig. 9-Polar diagram of the electric field strength for a small homogeneous ring quasi array of tangential or radial dipoles for various values of $H$.

polarized in this direction. For $|H| \geqq 2$ the field will have the same character for any value of $H$; however, the field concentrates to an increasing degree around the horizontal plane for increasing $|H|$. The quotient between the maximum values of the $\theta$ - and the $\varphi$-components of the electric field strength is expressed approximately by

$$
\frac{\left|F_{\theta}\right|_{\max }}{\left|F_{\varphi}\right|_{\mathrm{max}}} \cong \frac{1}{\sqrt{e(|H|-1)}},
$$

where $e$ is the base of the natural logarithms. Accordingly, with increasing $|H|$ the field will to an increasing degree become purely horizontally polarized in any direction, as is also seen from Fig. 9.

With an increasing radius of the ring quasi-array the field will to an increasing degree deviate from the field occurring in the case of an infinitely small radius of the 
quasi-array. However, even in the case of a radius so large as the minimum radius that can be chosen in order to avoid super-gain, the shape of the field will deviate little from the shape occurring in the case of an infinitely small radius.

In the above calculations we have assumed that the number of elements is infinitely large; the ring quasiarray was then exactly azimuthally omnidirectional. Apart from the case of $H=0$ and a very small radius, i.e., a small circular frame aerial with a constant current, the ring quasi-array must, however, in practice be composed of a finite number of elements. In order that the field from such a homogeneous ring quasi-array with a finite number of elements may be a fairly good approximation to the field from the corresponding quasi-array with infinitely many elements, the terms corresponding to $q=0$ in the expressions for $F_{\theta}$ and $F_{\varphi}$ must be the principal terms in the infinite series. The approximate condition that this will occur is that

$$
s>2|H| \text {. }
$$

Assuming that this inequality is satisfied, we may generally express the field with a sufficiently good approximation by including the first correction term. So doing we find

$$
\begin{gathered}
F_{\theta} \cong \cos \theta\left\{(-i)^{H} \frac{H}{z} J_{H}(z)\right. \\
+(-i)^{s}\left[(-i)^{H} e^{i s \phi} \frac{H+s}{z} J_{H+s}(z)\right. \\
\left.\left.-(-i)^{-H} e^{-i s \phi} \frac{-H+s}{z} J_{-H+s}(z)\right]\right\} e^{i H_{\phi}} \\
F_{\phi} \cong i\left\{(-i)^{H} J^{\prime}{ }_{H}(z)+(-i)^{s}\left[(-i)^{H} e^{i s \varphi} J_{H+s}^{\prime}(z)\right.\right. \\
\left.\left.+(-i)^{-H} e^{-i s_{\phi} J^{\prime}-H+s}(z)\right]\right\} e^{i H \theta} .
\end{gathered}
$$

In analogy with what we did in the case of the homogeneous ring array we shall here express the irregularity introduced into the field as a consequence of the fact that the number of elements is finite, by stating the relative, maximum variation of $F_{\theta}$ and $F_{\varphi}$. These relative, maximum variations are calculated on the basis of the expressions derived here analogously to the variations in the case of the homogeneous ring array. The description of this calculation is omitted here. The relative, maximum variation $\Delta\left|F_{\theta}\right|$ of the $\theta$-component of the electric field strength for $|H|=4$ is plotted in Fig. 10, and the relative, maximum variation $\Delta\left|F_{\varphi}\right|$ of the $\varphi$-component of the electric field strength for $H=0$, and $|H|=4$ is plotted in Fig. 11(a) and 11(b). Also in the case of a homogeneous ring quasi-array of tangential dipoles the case of $H=0$ is seen to exhibit the characteristic feature that 3 antennas give a better polar diagram than 4 , and 5 antennas a better diagram than 6 , etc.

\section{Radiation Resistance and Gain}

We shall here calculate the radiation resistance and the gain of a homogeneous ring quasi-array of tangential dipoles by using the Poynting vector method. For the sake of simplicity we shall confine ourselves to dealing

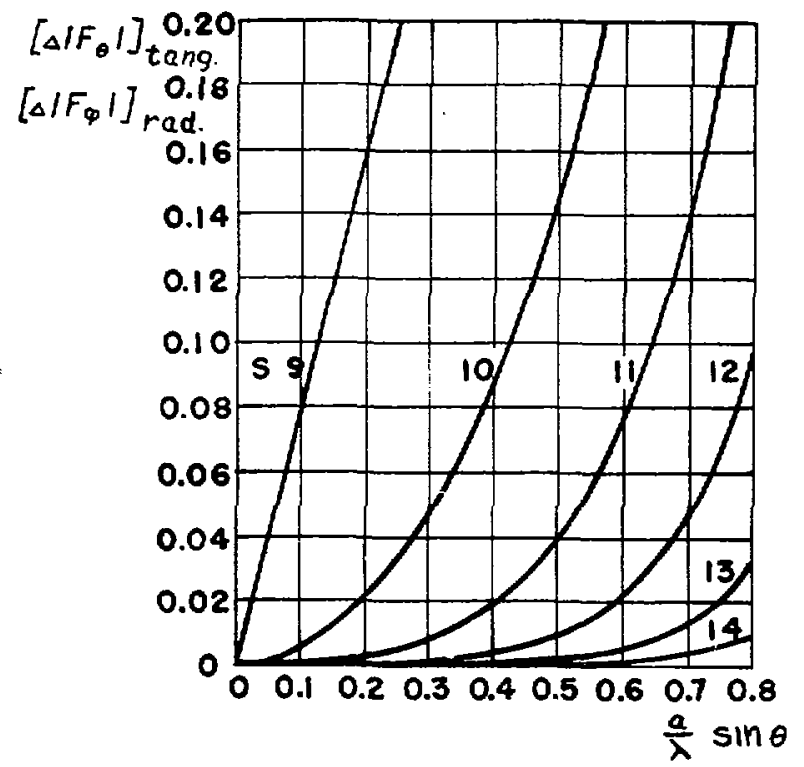

Fig. 10-The relative, maximum variation of one of the field components for a homogeneous ring quasi-array of tangential dipoles $\left(\Delta \mid F_{\theta} \vdots\right)$ or radial dipoles $\left(\Delta\left|F_{\phi}\right|\right)$ for $|H|=4$.

with the case where the number of dipoles is infinitely large; in practice, the result obtained in this way will apply with a good approximation also when the number of dipoles is finite.

Denoting the intrinsic impedance of space by $\xi=$ $\sqrt{\frac{\mu}{\epsilon}}$, we may express the Poynting vector $\bar{S}$ for an antenna with the normalization constant $K$ and the normalized electric field strength $\bar{F}(\theta, \varphi)$ by

$$
\bar{S}=\frac{\eta}{2}|\bar{E}| 2 \hat{r}=\frac{\eta|K|^{2}}{2 r^{2}} m(\theta, \varphi) \hat{r},
$$

where $\hat{r}$ denotes a unit vector pointing in the direction of the $r$-axis, and where the function $m(\theta, \varphi)$ is defined by

$$
m(\theta, \varphi)=\left|F_{\theta}\right|^{2}+\left|F_{\varphi}\right|^{2} .
$$

The effect $P$ radiated through a spherical surface having its center at the origin and the radius $r$, is expressed by

$$
P=\int_{0}^{\pi} \int_{0}^{2 \pi} \operatorname{Re}(\bar{S} \cdot \hat{r}) r^{2} \sin \theta d \theta d \varphi=2 \pi \eta|K|^{2} \mathrm{M},
$$

where the constant $M$ is defined by

$$
M=\frac{1}{4 \pi} \int_{0}^{\pi} \int_{0}^{2 \pi} m(\theta, \varphi) \sin \theta d \theta d \varphi .
$$

If $\left|F_{\theta}\right|$ and $\left|F_{\varphi}\right|$ and consequently also $m(\theta, \varphi)$ are independent of $\varphi$, as is the case with the ring quasi-array we are considering here, we have

$$
M=\frac{1}{2} \int_{0}^{\pi} m(\theta, \varphi) \sin \theta d \theta .
$$

The radiation resistance $R$ is defined as the resistance through which a certain reference current $I^{\prime}$ must flow in order that an effect equal to the radiated effect $P$ may be developed,

$$
P=\frac{1}{2} R\left|I^{\prime}\right|^{2}
$$

Consequently we find

$$
R=N M,
$$




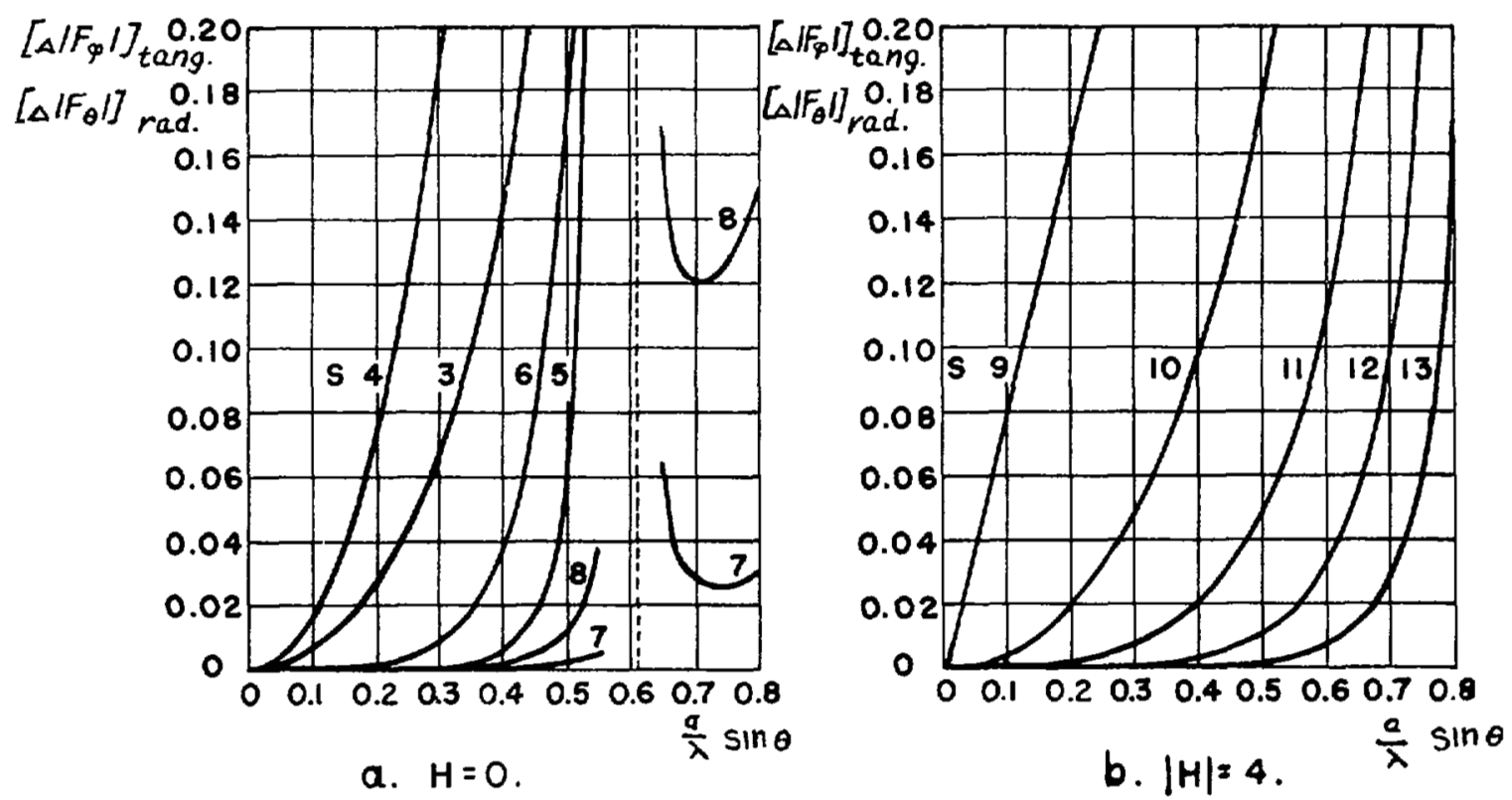

Fig. 11-The relative, maximum variation of one of the field components for a homogeneous ring quasi-array of tangential dipoles $\left(\Delta\left|F_{\phi}\right|\right)$ or radial dipoles $\left(\Delta\left|F_{\theta}\right|\right)$ for (a) $H=0$ and (b) $|H|=4$.

where

$$
N=\frac{4 \pi \eta|K|^{2}}{\left|I^{\prime}\right|^{2}}
$$

The gain $g(\theta, \varphi)$ of the antenna in a certain direction $(\theta, \varphi)$ may be expressed by the parameters defined above, the function $m(\theta, \varphi)$ and the constant $M$. We find

$$
g(\theta, \varphi)=\frac{|\bar{S}|}{\left.|\bar{S}|\right|_{\text {mean }}}=\frac{m(\theta, \varphi)}{M} .
$$

Accordingly, the radiation resistance $R$ as well as the gain $g(\theta, \varphi)$ of an antenna are determined by the constants $N$ and $M$ and the function $m(\theta, \varphi)$.

As the reference current $I^{\prime}$ for the homogeneous ring quasi-array in question we shall use the current $I$ in one of the elements, no matter which of them. The constant $N$ is then defined by

$$
N=\frac{(k s L)^{2} \zeta}{4 \pi} \text {. }
$$

The function $m(\theta, \varphi)$ is expressed by

$$
m(\theta, \varphi)=\left(\frac{H}{z} J_{H}(z) \cos \theta\right)^{2}+\left(J_{H}^{\prime}(z)\right)^{2}
$$

whereas the constant $M$ calculated by the use of this expression is given by

$$
M=\frac{1}{2} \int_{0}^{\pi}\left[\left(\frac{H}{z} J_{H}(z) \cos \theta\right)^{2}+\left(J^{\prime}{ }_{H}(z)\right)^{2}\right] \sin \theta d \theta .
$$

It may be shown that this integral may be expressed by known, tabulated functions and by the function $\bar{J}_{n}(x)=$ $\int_{a}^{x} J_{n}(t) d t$ for any value of $H$. In the case of $H=0$ this was demonstrated already by Foster. ${ }^{21}$ Consequently, we confine ourselves to carrying through the calculation for $|H| \geqq 1$. Through a simple transformation we find

$$
M=1 / 8\left[A_{H-1}(k a)+A_{H+1}(k a)-2 B_{H}(k a)\right],
$$

where the functions $A_{n}(x)$ and $\mathcal{B}_{n}(x)$ are defined by

$$
\begin{aligned}
& A_{n}(x)=\int_{o}^{\pi} J_{n}^{2}(x \sin \theta)\left(2-\sin ^{2} \theta\right) \sin \theta d \theta, \\
& B_{n}(x)=\int_{0}^{\pi} J_{n-1}(x \sin \theta) J_{n+1}(x \sin \theta) \sin ^{3} \theta d \theta .
\end{aligned}
$$

As shown in an earlier publication these integrals may be expressed in the following way ${ }^{1}$

$$
\begin{aligned}
A_{n}(x)=\left(\frac{3}{2 x}-\right. & \left.\frac{4 n^{2}-1}{8 x^{3}}\right) \bar{J}_{2 n}(2 x) \\
& +\frac{2 n-1}{4 x^{2}} \bar{J}_{2 n}(2 x)-\frac{1}{2 x} J_{2 n+1}(2 x) \\
B_{n}(x)= & \left(-\frac{1}{2 x}+\frac{3\left(4 n^{2}-1\right)}{8 x^{3}}\right) J_{2 n}(2 x) \\
& -\frac{3(2 n-1)}{4 x^{2}} J_{2 n}(2 x)+\frac{3}{2 x} J_{2 n+1}(2 x) .
\end{aligned}
$$

For the case of $n=0$ several tables of the function $\bar{J}_{n}(x)$ occurring here have been published; the most comprehensive of these tables seems to be the one calculated by Lowan and Abramowitz. ${ }^{23}$ On the basis of this table the author has calculated a table of $J_{n}(x)$ for $n=1,2 \cdots, 8$ and for $x=0(0.01) 10 .{ }^{1}$ The values of the function are given to five places of decimals; there is an inaccuracy of \pm 1 in the last decimal place.

From the expressions for $N, m(\theta, \varphi)$, and $M$ obtained here we can now easily calculate the radiation resistance $R$ and the gain $g(\theta, \varphi)$ of the homogeneous ring quasiarray of tangential dipoles. The radiation resistance $R$

${ }^{23}$ A. N. Lowan and M. Abramowitz, "Table of the integrals" $\int_{o}^{x} J_{o}(t) d t$ and $\int_{o}^{x} Y_{o}(t) d t,{ }^{\prime}$ Jour. Math. Phys., vol, 22, pp. 2-12; 1943. 


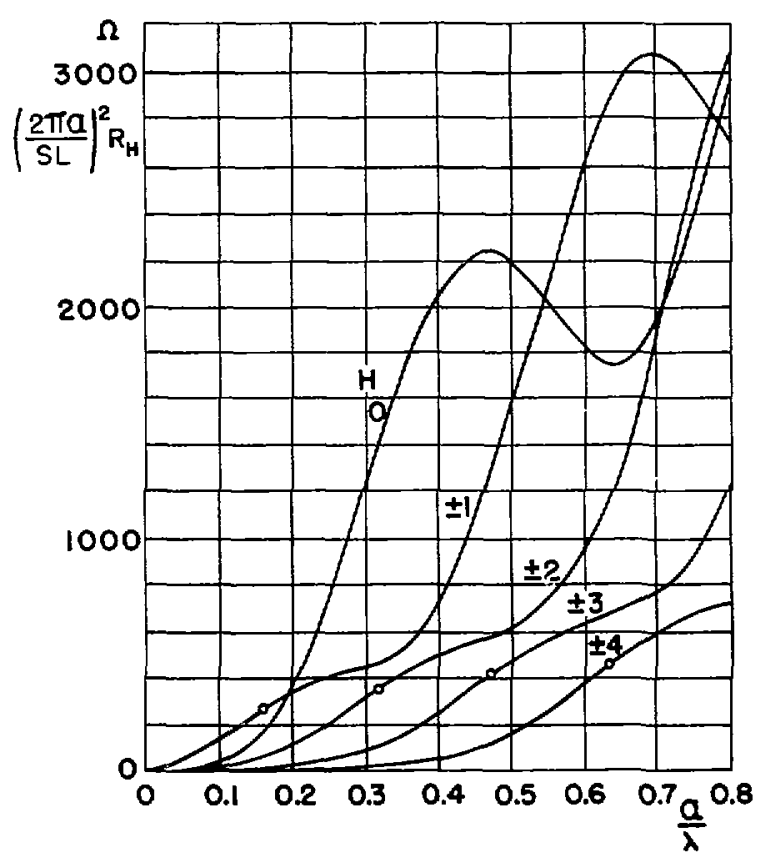

Fig. $12-(2 \pi a / s L)^{2}$ multiplied by the radiation resistance of a homogeneous ring quasi-array of tangential dipoles as a function of $a / \lambda$ for various values of $H$.

multiplied by $(2 \pi a / s L)^{2}$ is plotted in Fig. 12 as a function of $a / \lambda$ for various values of $H$. The radiation resistance is seen to decrease with increasing increment of the current phase and to increase, though not monotonically, with increasing radius. In Fig. 13 is plotted the gain $g(\theta, \varphi)$ as a function of $a / \lambda$ and for various values of $H$, partly for horizontal directions $\theta=\pi / 2$ and partly for vertical directions $\theta=0$ or $\pi$. Only the ring quasi-array with $|H|=1$ has in the vertical direction a gain that is different from zero.

\section{Homogeneous Ring Quasi-Array of Tangential, Linear} Antennas with a Constant Current.

In dealing with the homogeneous ring quasi-array of tangentially oriented dipoles we have so far assumed the elements to be Hertz dipoles; i.e., infinitely short dipoles. For many applications this was a good approximation, and it made the mathematical treatment rather simple so that in such cases where this approximation was permissible, the principally important facts were emphasized more than would have been the case if less idealizing assumptions had been made. In practice, however, a ring quasi-array of the type considered here must be composed of antennas of finite dimensions, e.g., of a number of linear wire antennas. For the simple case where all currents are in the same phase; i.e., for $H=0$, such a design has been suggested by Moullin. ${ }^{22}$ However, if the current distribution on the various antennas is known, it is possible to carry out a calculation of the radiated field along the same lines as we did above in calculating the field from a homogeneous ring quasi-array of Hertz dipoles. The field will then be expressed by a principal term that is independent of the azimuth, in addition to correction terms dependent on the azimuth and originating from the finite number of elements. As an example of such a field calculation we shall here give the expression for the field from a homogeneous ring quasi-array of linear wire antennas with a constant current. As a special case of this we shall obtain the expression for the field from a polygonal frame aerial with a constant current.

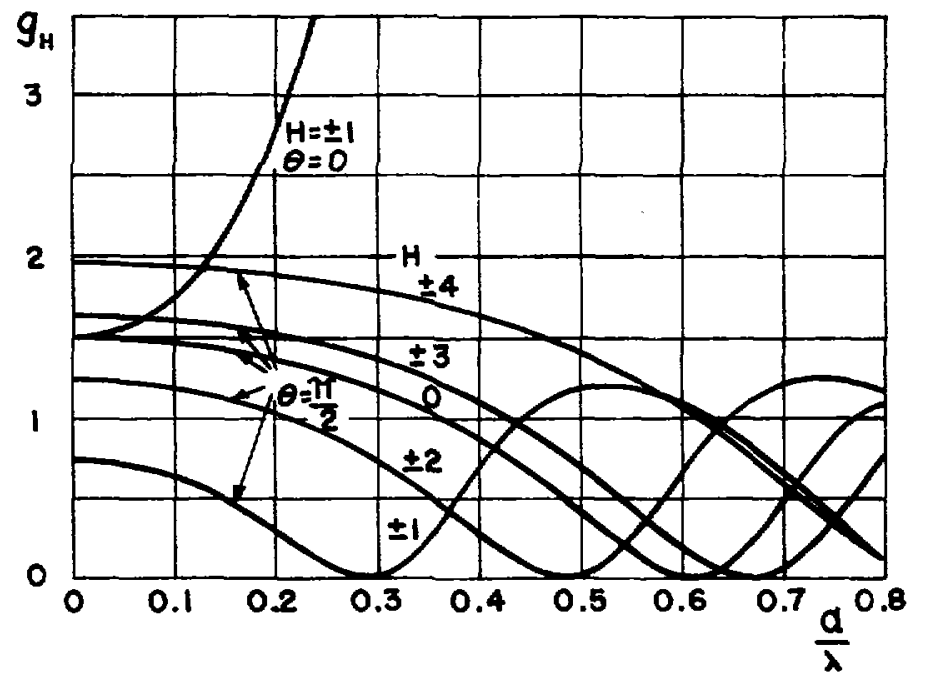

Fig. 13-The gain in horizontal and vertical directions for a homogeneous ring quasi-array of tangential dipoles as a function of $a / \lambda$ for various values of $H$.

We consider a ring quasi-array of $s$ tangentially oriented, linear antennas having the same length $l$. The mid-points of the antennas are assumed to be placed equidistantly along the periphery of a circle with radius $a$, as shown in Fig. 14. The antenna $j$ will thus coincide

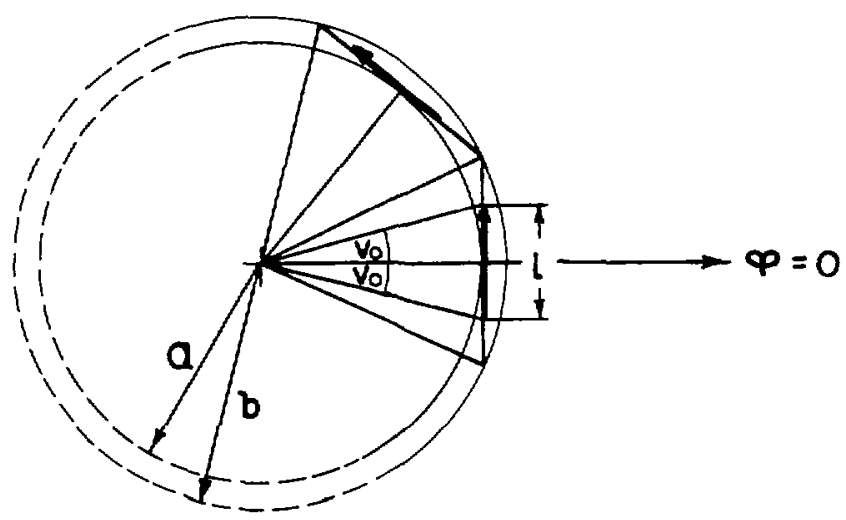

Fig. 14-Ring quasi-array of $s$ tangentially oriented linear antennas of length $l$.

with the side $j$ in a regular $s$-angle, the inscribed circle of which is the said circle with radius $a$. A spherical co-ordinate system is introduced as shown in the figure so that the mid-point of antenna $j$ has the co-ordinates $\left(a, \pi / 2, u_{j}\right)$, where

$$
u_{j}=\frac{2 \pi j}{s} \quad j=1,2, \cdots, s .
$$

The current $I_{j}$ in antenna $j$ is supposed to be given by

$$
I_{j}=I e^{i H u_{j}}
$$


In analogy with the calculations previously carried out we introduce a normalizing constant $K$ defined by

$$
K=\frac{i k s l \zeta I}{4 \pi}
$$

the field will then be determined by the specification of the normalized electric field strength $\vec{F}(\theta, \varphi)$ corresponding to this constant.

In order to be able to express $F$ in a simple way we define the following functions;

$$
\begin{aligned}
& P_{n, r}(x)=\frac{1}{\operatorname{tg} r} \int_{0}^{r} J_{n}\left(\frac{x}{\cos v}\right) \frac{\cos n v}{\cos ^{2} v} d v, \\
& P s_{n, r}(x)=\frac{1}{2}\left[P_{n-1, r}(x)+P_{n+1, r}(x)\right] \\
& P d_{n, r}(x)=\frac{1}{2}\left[P_{n-1, r}(x)-P_{n+1, r}(x)\right] .
\end{aligned}
$$

By using these functions we may express the components of the normalized electric field strength in the following way

$$
\begin{array}{r}
F_{\theta}=\frac{1}{2} \cos \theta \sum_{q=0}^{\infty}\left(2-\delta_{o q}\right)\left[P s_{H+q s, v_{0}}(z) e^{i(H+q s)(\phi-\pi / 2)}\right. \\
\left.-P S_{-H+q s, v_{o}}(z) e^{-i(-H+q s)(\phi+\pi / 2)}\right] \\
F_{\phi}=\frac{i}{2} \sum_{q=0}^{\infty}\left(2-\delta_{o q}\right)\left[P d_{H+q s, v_{o}}(z) e^{i(H+q s)(\dot{\phi}-\pi / 2)}\right. \\
\left.\quad+P d_{-H+q s, v_{o}}(z) e^{-i(-H+q s)(\phi+\pi / 2)}\right]
\end{array}
$$

where

$$
z=k a \sin \theta
$$

and

$$
v_{0}=\operatorname{arctg} \frac{l}{2 a} .
$$

It is seen that this expression may be derived from the expression for the field from a homogeneous ring quasiarray of tangential Hertz dipoles given above by replacing $n / z J_{n}(z)$ and $J^{\prime}{ }_{n}(x)$ by $P s_{n, v_{o}}(x)$ and $P d_{n, v_{o}}(x)$ respectively. When the length $l$ of the antennas converges towards zero, the expressions for the field components derived here converge towards the expressions for the field components of a corresponding quasi-array of Hertz dipoles.

In the case where the number $s$ of the elements is infinitely large we find from the expressions given above the following expressions for the field components,

$$
\begin{gathered}
F_{\theta}=\cos \theta P s_{H}, v_{o}(z) e^{i H(\phi-\pi / 2)}, \\
F_{\phi}=i P d_{H, v_{o}}(z) e^{i H(\phi-x / 2)} .
\end{gathered}
$$

For a homogeneous ring quasi-array of antennas with a sine-shaped current distribution corresponding expressions for the field may be derived; the functions which must be introduced in this case are more complicated than those occurring here.

\section{Homogeneous Ring Quasi-ARray of Radial Antennas}

Homogeneous Ring Quasi-Array of Radial Hertz Dipoles Radiated Field; In the preceding section we have seen that a homogeneous ring quasi-array of tangential antennas radiates an essentially horizontally polarized field which to an increasing degree concentrates around the horizontal plane with increasing increment of the current phase per revolution. It may be supposed that a homogeneous ring quasi-array of radial dipoles radiates a field of a similar type. We shall therefore carry out an investigation of the field from such an antenna system.

Certain types of homogeneous ring quasi-array have long since found practical application. Böhm has suggested an antenna with reduced radiation for high elevation angles, and which consists of a vertical wire being connected at its upper end to a system of radial wires of such a length that regard has to be taken of the radiation from these wires. ${ }^{12}$ The radiation from the radial wires results in a reduction of the field radiated at high elevation angles so that the antenna may be used as a fading-reducing antenna. Böhm ${ }^{24}$ has calculated the field radiated by the radial wires on the assumption that the number of wires is infinitely large, so that they form a disk, and so that the total current passing any circle concentric with the periphery of the disk is constant. Whereas this antenna is composed of radial wires with currents in the same phase, the turnstile antenna invented by Brown ${ }^{25}$ may be considered constructed by radial wires with currents the phase of which increases $2 \pi$ during one revolution. Thus, with the notation used above, these antennas correspond to $H=0$ and $H=1$ respectively.

As the simplest type of a homogeneous ring quasiarray of radial antennas we shall here consider such a ring quasi-array of radial Hertz dipoles. For the special values of $H, H=0$ and $|H|=1$, the field from this quasi-array was previously calculated by Carter. ${ }^{15}$ We extend the investigation of the ring quasi-array in question to quasi-arrays with an arbitrary value of $H$, so that the possibility of arriving at new, usable antenna systems of this type is explored.

We shall calculate the field from the ring quasi-array shown in Fig. 15, of $s$ radially oriented Hertz dipoles of the length $L$ placed equidistantly along a circle with radius $a$. A spherical co-ordinate system $(r, \theta, \varphi)$ is introduced so that the co-ordinates of the $j$ th dipole will be $\left(a, \pi / 2, u_{j}\right)$, where

$$
u_{j}=\frac{2 \pi j}{s} \quad j=1,2, \cdots, s .
$$

The current $I_{j}$ in the $j$ th dipole is assumed to be

$$
I_{j}=I e^{i H u_{j}} \text {. }
$$

The calculation of the field is carried out in the same manner as for a homogeneous ring quasi-array of tangential dipoles. Introducing the normalization constant $K$, defined by

$$
K=\frac{i k s L \zeta I}{4 \pi}
$$

24 O. Böhm, "Rundfunk-Sendeantennen mit vertikal gebündelter Ausstrahlung," Hochfreq. u. Elektroak., vol. 42, pp. 137-145; 1933.

${ }^{25}$ G. H. Brown, "Turnstile aerials," Electronics, vol. 9, pp. 14-17, 48; April, 1936. 
we find the following expressions for the components of the normalized electric field strength $\overline{F(} \theta, \phi)$;

$$
\begin{gathered}
F_{\theta}=\frac{i}{2} \cos \theta \sum_{q=o}^{\infty}\left(2-\delta_{o q}\right)\left[J_{H+q s}^{\prime}(z) e^{i(H+q s)(\phi-\pi / 2)}\right. \\
\left.\quad+J_{-H+q \theta}^{\prime}(z) e^{-i(-H+q s)(\phi+\pi / 2)}\right], \\
F_{\phi}=-\frac{1}{2} \sum_{q=0}^{\infty}\left(2-\delta_{o q}\right)\left[\frac{H+q s}{z} J_{H+q s}(z) e^{i(H+q s)(\phi-\pi / 2)}\right. \\
\left.-\frac{-H+q s}{z} J_{-H+q s}(z) e^{-i(-H+q s)(\phi+\pi / 2)}\right]
\end{gathered}
$$

where

$$
z=k a \sin \theta
$$

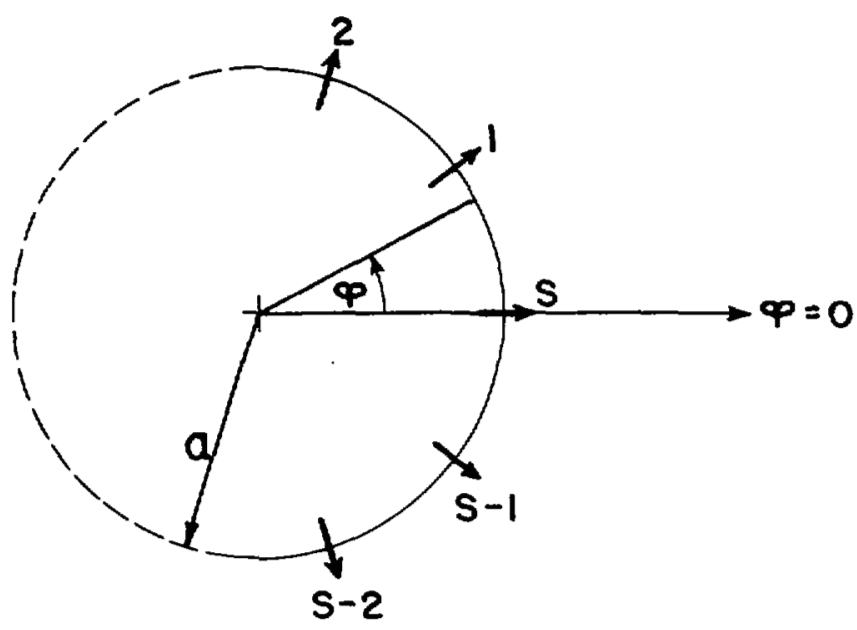

Fig. 15-Ring quasi-array of $s$ radial dipoles.

We shall first consider the field in the case where the number of elements is infinitely large. From the above expression we find, for $s=\infty$,

$$
\begin{aligned}
& F_{\theta}=i J_{H}^{\prime}(z) \cos \theta e^{i H(\phi-\pi / 2)}, \\
& F_{\varphi}=-\frac{H}{z} J_{H}(z) e^{i H(\phi-x / 2)} .
\end{aligned}
$$

The radiated field will thus in general be elliptically polarized. Only for $H=0$ the $\varphi$-component of the field strength is zero in every direction so that the field is polarized in the direction of the $\theta$-axis. For $|H| \geqq 1$ points in the horizontal plane having the same phase will be situated on an Archimedes' spiral just as in the case of the homogeneous ring arrays and ring quasiarrays considered earlier in this paper.

A general idea of the structure of the field for various values of $H$ is best obtained by investigating the field for an infinitely small radius of the ring quasi-array. From the expressions given above we find for $k a \ll 1$

$$
\begin{aligned}
& F_{\theta}= \begin{cases}-i \frac{k a}{2} \cos \theta \sin \theta & \text { for } \mathrm{H}=0, \\
i \frac{(k a)^{H-1}}{2^{B}(H-1) !} \cos \theta \sin H^{H-1} \theta e^{i H(\phi-\pi / 2)} & \text { for }|H| \geqq 1,\end{cases} \\
& F_{\varphi}= \begin{cases}0 & \text { for } H=0, \\
-\frac{(k a)^{H-1}}{2^{B}(H-1) !} \sin ^{H-1} \theta e^{i I I(\phi-\pi / 2)} & \text { for }|H| \geqq 1 .\end{cases}
\end{aligned}
$$

Fig. 9(b) shows the field for $H=0$. In the case of $|H| \geqq 1$ the field from the ring quasi-array considered here is seen to be identical with the field from the ring quasi-array of tangential Hertz dipoles previously investigated. For $|H|=1,2$, and 9 this field is plotted in Fig. 9(c)-9(e). The comments made on the ring quasi-array of tangential dipoles are thus applicable here, too.

It is only for an infinitely small radius and for $|H| \geqq 1$ that the field from a homogeneous ring quasi-array of radial dipoles is identical with the field from a corresponding homogeneous ring quasi-array of tangential dipoles. With an increasing radius of the ring quasi-array of radial dipoles considered here the radiated field will deviate to an increasing degree from the ideal field shown in Fig. 9 as well as from the field radiated from a corresponding ring quasi-array of tangential dipoles.

So far we have assumed that the number of elements is infinitely large. In practice, however, the number will be finite, and we must then use the complete expression for the field given earlier. We suppose that the number $s$ of elements is larger than $2|H|$,

$$
s>2|H| \text {. }
$$

The term corresponding to $q=0$ in the expressions for $F_{\theta}$ and $F_{\varphi}$ will then be the dominant term, and we may therefore express the field components by including only one extra term in each of the infinite series

$$
\begin{gathered}
F_{\theta} \cong i \cos \theta\left[J_{H}^{\prime}(z) e^{i H(\phi-\pi / 2)}+J_{H+s}^{\prime}(z) e^{i(H+s)(\phi-\pi / 2)}\right. \\
\left.+J_{-H+s}^{\prime}(z) e^{-i(-H+s)(\phi+\pi / 2)}\right], \\
F_{\phi} \cong-\left[\frac{H}{z} J_{H}(z) e^{i H(\phi-\pi / 2)}+\frac{H+s}{z} J_{H+s}(z) e^{i(H+s)(\phi-\pi / 2)}\right. \\
\left.-\frac{-H+s}{z} J_{-H+8}(z) e^{-i(-H+s)(\phi+\pi / 2)}\right] .
\end{gathered}
$$

It appears from these expressions that the relative maximum variation $\Delta\left|F_{\theta}\right|$ and $\Delta\left|F_{\varphi}\right|$ for the quasiarray considered here is equal to $\Delta\left|F_{\phi}\right|$ and $\Delta\left|F_{\theta}\right|$ respectively for the corresponding homogeneous ring quasi-array of tangential dipoles previously dealt with. Consequently Fig. $11(\mathrm{a})$ and $11(\mathrm{~b})$ show the relative maximum variation of the $\theta$-component for a homogeneous ring quasi-array with radially oriented elements for $H=0$ and $|H|=4$, and Fig. 10 the relative, maximum variation of the $\varphi$-component for such an array for $|H|=4$.

\section{Radiation Resistance and Gain}

We shall now calculate the radiation resistance and the gain for a homogeneous ring quasi-array of infinitely many Hertz dipoles. To a good approximation the results hereby obtained will also be applicable to a ring quasiarray with a finite number of elements when the number of elements is as large as the considerations to be taken for obtaining a fair approximation to an azimuthally omnidirectional antenna system permit. The calculation is made in close analogy to the corresponding calculation for a homogeneous ring quasi-array of tangential Hertz dipoles. 
It being assumed that the radiation resistance $R$ is given by the current in an odd dipole as a reference current, the constant $N$ previously introduced will be

$$
N=\frac{(k s L)^{2} \zeta}{4 \pi} \text {. }
$$

Hereby the function $m(\theta, \varphi)$ will be expressed by

$$
m(\theta, \varphi)=\left(J^{\prime}{ }_{H I}(z) \cos \theta\right)^{2}+\left(\frac{H}{z} J_{H}(z)\right)^{2},
$$

whereas the constant $M$ calculated from this expression is given by

$$
M=\frac{1}{2} \int_{0}^{\pi}\left[\left(J_{H}^{\prime}(z) \cos \theta\right)^{2}+\left(\frac{H}{z} J_{H}(z)\right)^{2}\right] \sin \theta \mathrm{d} \theta .
$$

We shall here confine ourselves to giving the expression for the radiation resistance for $|H| \geqq 1$. It may then be shown that the expression for $M$ may be transformed into

$$
M=\frac{1}{8}\left[A_{H-1}(k a)+A_{H+1}(k a)+2 B_{H}(k a)\right],
$$

where $A_{n}(x)$ and $B_{n}(x)$ are the functions defined in a preceding section. From $N, m(\theta, \varphi)$, and $M$ we may now calculate the radiation resistance and the gain. The radiation resistance $R$ multiplied by $(2 \pi a / s L)^{2}$ is plotted in Fig. 16 as a function of $a / \lambda$ for the various values of

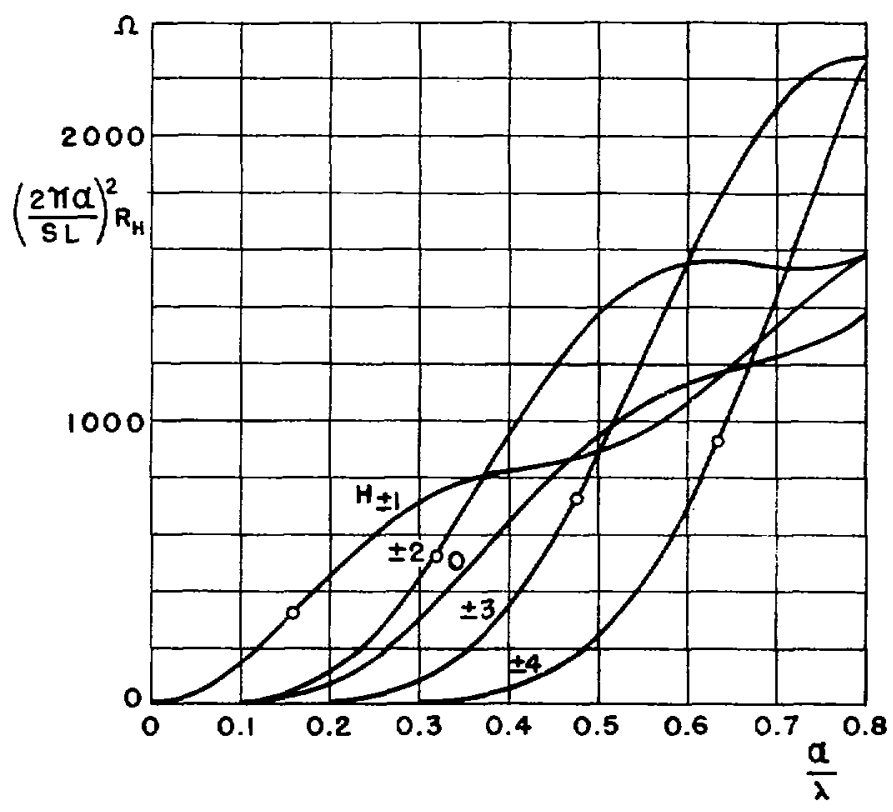

Fig. 16- $(2 \pi a / s L)^{2}$ multiplied by the radiation resistance of a homogeneous ring quasi-array of radial dipoles as a function of $a / \lambda$ for various values of $H$.

$H$, whereas the gain $g(\theta, \varphi)$ is plotted in Fig. 17. The curves showing the radiation resistance and the gain of the ring quasi-array investigated here are seen to have a shape similar to, but quantitatively deviating from, the corresponding curves for the ring quasi-array of tangentially oriented dipoles previously investigated.

Homogeneous Ring Quasi-Array of Radial, Linear Antennas with a Sine-Shaped Current

In the investigation made above of homogeneous ring quasi-arrays of radially oriented dipoles we have so far assumed, for the matter of simplicity, that the elements of the ring quasi-arrays were Hertz dipoles. In practice, however, the quasi-arrays will be composed of linear antennas having a finite length. This applies to such antennas as the disk antenna suggested by $\mathrm{Böhm}^{24}$ and the turnstile antenna suggested by Brown, ${ }^{25}$ and it will apply also to ring quasi-arrays for larger values of $|H|$.

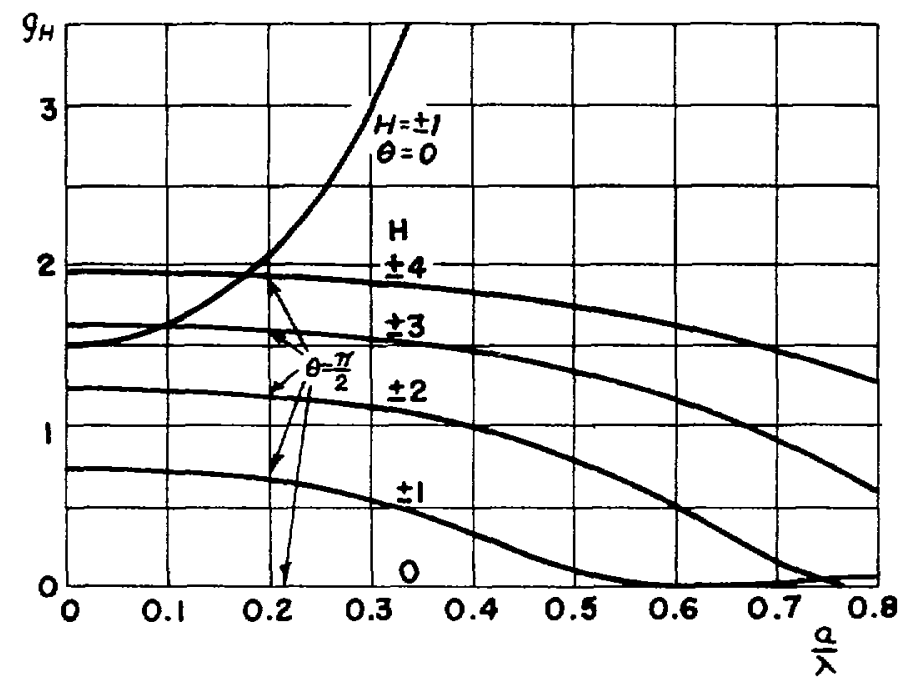

Fig. 17-The gain in horizontal and vertical directions for a homogeneous ring quasi-array of radial dipoles as a function of $a / \lambda$ for various values of $H$.

When the current distribution on the linear antennas is known, it is possible to derive an expression for the field similar to the expression derived above for the field from a ring quasi-array of radial Hertz dipoles.

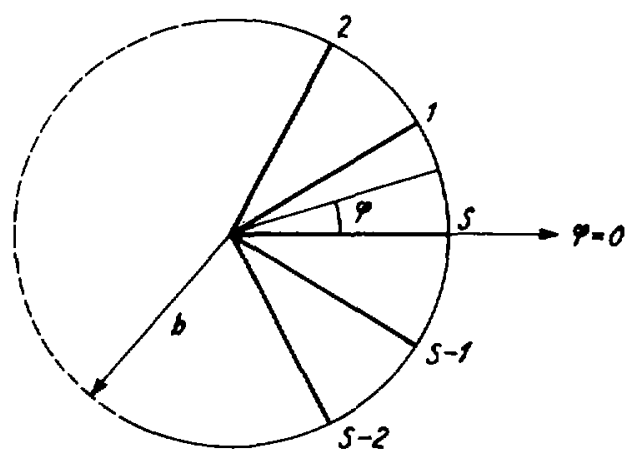

Fig. 18-Ring quasi-array of a radially oriented, linear antenna.

By way of example we shall here give the field from a ring quasi-array of $s$ linear antennas of the length $b$, placed as the spokes of a wheel, as shown in Fig. 18, and carrying sine-shaped currents $I_{j}$ of the same numerical value. The phase of these currents increases from one antenna to another so that its increment during one revolution amounts to $H 2 \pi$. With the notation introduced in Fig. 18 the current $I_{j}$ in the current maximum (actual or virtual) of antenna $j$ may be expressed by

where

$$
I_{j}=I e^{i H}{ }_{j},
$$

$$
u_{j}=\frac{2 \pi j}{s} \quad j=1,2, \cdots, s .
$$


Introducing a spherical co-ordinate system $(r, \theta, \varphi)$ in the usual way and defining the normalization constant

$$
K=\frac{i k s b \zeta I}{4 \pi},
$$

we may express the components of the normalized electric field strength $\bar{F}(\theta, \varphi)$ by

$$
\begin{array}{r}
F_{\theta}=\frac{i}{2} \frac{\cos \theta}{k b} \sum_{q=0}^{\infty}\left(2-\delta_{o q}\right)\left[p d_{H+q s, \theta}(k b) e^{i(H+q s)(\phi-\pi / 2)}\right. \\
\left.+p d_{-H+q s, \theta}(k b) e^{-i(-H+q s)(\phi+\pi / 2)}\right], \\
F_{\varphi}=-\frac{1}{2} \frac{1}{k b} \sum_{q=0}^{\infty}\left(2-\delta_{o q}\right)\left[p s_{H+q s, \theta}(k b) e^{i(H+q s)(\phi-\pi / 2)}\right. \\
-p s_{-H+q s, \theta}(k b) e^{-i(-H+q s)(\phi+\pi / 2)} .
\end{array}
$$

The functions $p s_{n, \theta}(x)$ and $p d_{n, \theta}(x)$ occurring in these expressions are defined by

where

$$
\begin{aligned}
& p s_{n, \theta}(x)=\frac{1}{2}\left[p_{n-1, \theta}(x)+p_{n+1, \theta}(x)\right], \\
& p d_{n, \theta}(x)=\frac{1}{2}\left[p_{n-1, \theta}(x)-p_{n+1, \theta}(x)\right],
\end{aligned}
$$

$$
p_{n, \theta}(x)=\int_{0}^{x} \sin (x-t) J_{n}(t \sin \theta) d t .
$$

It seems impossible generally to express this function in a simple way by known functions. However, an exception is the important case $\theta=\pi / 2$ corresponding to radiation in the horizontal plane $\theta=\pi / 2$. As shown by the author, we have

$$
\int_{0}^{x} \sin (x-t) J_{n}(t) d t=\left\{\begin{array}{l}
(-1)^{n / 2}\left[n \sin x-x J_{0}(x)\right. \\
+2 \sum_{j=1}^{\frac{n-1}{2}}(-1)^{i}(n+1-2 j) \\
\left.J_{2 j-1}(x)\right] \quad \text { for } n \text { odd } \\
(-1)^{n / 2}\left[n\left(\cos x-J_{o}(x)\right)\right. \\
+x J_{1}(x)+2 \sum_{j=1}^{n / 2-1}(-1)^{j+1} \\
\left.(n-2 j) J_{2 j}(x)\right] \text { for } n \text { even. }
\end{array}\right.
$$

For $H=1, s=4$, and $b=\lambda / 4$ the above formulas will express the field from an ordinary turnstile antenna.

\section{Homogenous Ring Quasi-ARRay of Oblique ANTENNAS}

In the investigations made until now of homogeneous ring arrays and ring quasi-arrays of dipoles we have assumed that the dipoles were axial, tangential, or radial; we have hereby obtained knowledge of and a basis for the design of some useful types of antennas. However, it is conceivable that some types of ring quasiarrays of obliquely oriented antennas may also turn out to be useful for practical purposes. An antenna system which in principle is a special case of such a ring quasiarray, is the antenna system invented by Lindenblad, ${ }^{26}$ which has been further investigated by Brown and Woodward;27 with the notation used here it may be

${ }^{26}$ N. E. Lindenblad, "Antennas and transmission lines at the Empire State Television Station. Part 2, "Communicalions, vol. 21, pp. 10-14, 24-26; April, 1941.

${ }^{27} \mathrm{G}$. H. Brown and O. M. Woodward, "Circularly-polarized omnidirectional antenna," $R C A R e v$. vol. 8, pp. 259-269; 1947. described as a homogeneous ring quasi-array, with $H=0$, of linear antennas that are perpendicular to the radius at their center, and that form a suitable angle with the horizontal plane, this angle depending on the radius of the circle. This antenna system radiates in horizontal directions a circularly polarized field that is independent of azimuth; for this reason it is suited for communication between a fixed station and an airplane.

We shall here generalize the investigation made by Brown and Woodward, setting ourselves the task of calculating the field from a homogeneous ring quasiarray with an arbitrary $H$ of an arbitrary number of dipoles with an arbitrary orientation. The antenna system treated by Brown and Woodward will then be comprised as a special case by this investigation, but the expression for the field will be presented here in a form more suitable as a basis for the design. The homogeneous ring arrays and ring quasi-arrays previously dealt with in this paper will also be included as special cases in the following investigation.

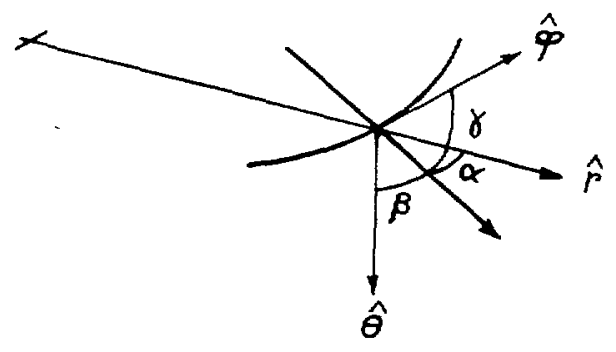

Fig. 19-Orientation of a dipole in a homogeneous ring quasiarray of oblique dipoles.

We consider $s$ Hertz dipoles of the length $L$ that are placed equidistantly along a circle with radius $a$, as shown in Fig. 1. The antennas are assumed to form the angles $\alpha, \beta$, and $\gamma$ with the $\gamma$-, $\theta-$, and $\varphi$-axes, respectively, in a spherical co-ordinate system, as shown in Fig. 19. The current in the $j$ th dipole is supposed to be given by

where

$$
I_{j}=I e^{i H u}{ }_{j}
$$

$$
u_{j}=\frac{2 \pi j}{s} \quad j=1,2, \cdots, s .
$$

With the normalization constant $K$ being defined by

$$
K=\frac{i k s L \zeta I}{4 \pi}
$$

the normalized electric field strength $\bar{F}(\theta, \varphi)$ may now be expressed by

$$
\begin{gathered}
F_{\theta}=\frac{1}{2} \sum_{q=o}^{\infty}\left(2-\delta_{o q}\right)\left\{\left[i J_{H+q s}^{\prime}(z) \cos \theta \cos \alpha\right.\right. \\
+J_{H+q s}(z) \sin \theta \cos \beta \\
\left.+\frac{H+q s}{z} J_{H+q s}(z) \cos \theta \cos \gamma\right] e^{i(H+q s)(\varphi-\pi / 2)} \\
+\left[i J_{-H+q s}^{\prime}(z) \cos \theta \cos \alpha\right.
\end{gathered}
$$




$$
\begin{aligned}
& +J_{-H+q s}(z) \sin \theta \cos \beta-\frac{-H+q s}{z} J_{-H+q s}(z) \\
& \left.\cos \theta \cos \gamma] e^{-i(-H+q s)(\varphi+\pi / 2)}\right\}, \\
& F_{\varphi}=\frac{1}{2} \sum_{q=o}^{\infty}\left(2-\delta_{o q}\right)\left\{\left[-\frac{H+q s}{z} J_{H+q s}(z) \cos \alpha\right.\right. \\
& \left.+i J^{\prime}{ }_{H+q s}(z) \cos \gamma\right] e^{i(H+q s)(\varphi-\pi / 2)} \\
& +\left[\frac{-H+q s}{z} J_{-H+q s}(z) \cos \alpha+i J_{-H+q s}^{\prime}(z) \cos \gamma\right] \\
& \left.e^{-i(-H+q s)(\varphi+\pi / 2)}\right\} \text {. }
\end{aligned}
$$

The terms corresponding to $q=0$ in these expressions denote the field corresponding to an infinitely large number of elements. Of the many possible antenna systems that may result from using different values of $\alpha, \beta, \gamma$, and $H$ we shall only give attention here to the following two important cases: $\alpha=\pi / 2, H=0$, and $\gamma=\pi / 2, H=1$.

$\alpha=\pi / 2, H=0$. In this case the dipoles are tangents to a circular cylinder. They form the angle $\gamma$ with the horizontal plane, and they carry currents with the same phase. If we choose especially

$$
\gamma= \pm \operatorname{arctg} \frac{J_{1}(k a)}{J_{o}(k a)}
$$

the field components in the case of an infinite number of elements will be expressed by

$$
\begin{gathered}
F_{\theta}= \pm \frac{J_{1}(k a)}{J_{o}(k a)} J_{0}(z) \sin \theta \cos \gamma, \\
F_{\varphi}=-i J_{1}(z) \cos \gamma .
\end{gathered}
$$

The antenna system designed in that way is seen to be azimuthally omnidirectional and in horizontal directions to radiate a circularly polarized field. In the vertical direction the field is zero. The quasi-array dealt with here is the very antenna system invented by Lindenblad $^{26}$ and further investigated by Brown and Woodward ${ }^{27}$ discussed previously.

When the ring quasi-array has such a small radius that $k a \ll 1$, we find

$$
\begin{aligned}
& F_{\theta} \cong \pm \frac{k a}{2} \sin \theta \\
& F_{\varphi} \cong-i \frac{k a}{2} \sin \theta .
\end{aligned}
$$

The field is then seen to be circularly polarized in any direction in space. The polar diagram for each of the field components becomes identical with the diagram shown in Fig. 3(a).

From the general expression given above for the field from a homogeneous ring quasi-array of an arbitrary number of oblique elements we easily find the relative, maximum variation of the antenna system considered here, when this system has a finite number of elements.

$\gamma=\pi / 2, H=1$. In this case the dipoles are placed along the edges of a pyramid; they make the angle $\alpha$ with the horizontal plane, and the current phases increase
$2 \pi$ during one revolution. The antenna system is azimuthally omnidirectional, and the field radiated in the vertical direction is circularly polarized. In horizontal directions the field will be linearly polarized. By choosing a suitable angle of inclination $\alpha$ for the antennas we may obtain that the field radiated in horizontal directions forms an angle of 45 degrees with the horizontal plane. We put

$$
\alpha= \pm \operatorname{arctg} \frac{1}{k a}
$$

and obtain hereby the following expressions for the field components in the case of an infinitely large number of antennas

$$
\begin{gathered}
F_{\theta}=\left[i J_{1}^{\prime}(z) \cos \theta \pm \frac{1}{k a} J_{1}(z) \sin \theta\right] \cos \left(\operatorname{arctg} \frac{1}{k a}\right) e^{i(\varphi-\pi / 2)}, \\
F_{\varphi}=-\frac{1}{z} J_{1}(z) \cos \left(\operatorname{arctg} \frac{1}{k a}\right) e^{i(\varphi-\pi / 2)} .
\end{gathered}
$$

In the vertical direction this field is circularly polarized. In horizontal directions it is linearly polarized, and the electric field strength extends an angle of \pm 45 degrees with the horizontal plane.

A general impression of the character of the field is best obtained by considering a ring quasi-array of the type considered here with such a small radius that $k a \ll 1$. We then have

$$
\begin{gathered}
F_{\theta} \cong \frac{1}{2} \cos \left(\operatorname{arctg} \frac{1}{k a}\right)\left[i \cos \theta \pm \sin ^{2} \theta\right] e^{i(\varphi-\pi / 2)}, \\
F_{\varphi} \cong-\frac{1}{2} \cos \left(\operatorname{arctg} \frac{1}{k a}\right) e^{i(\varphi-\pi / 2)}
\end{gathered}
$$

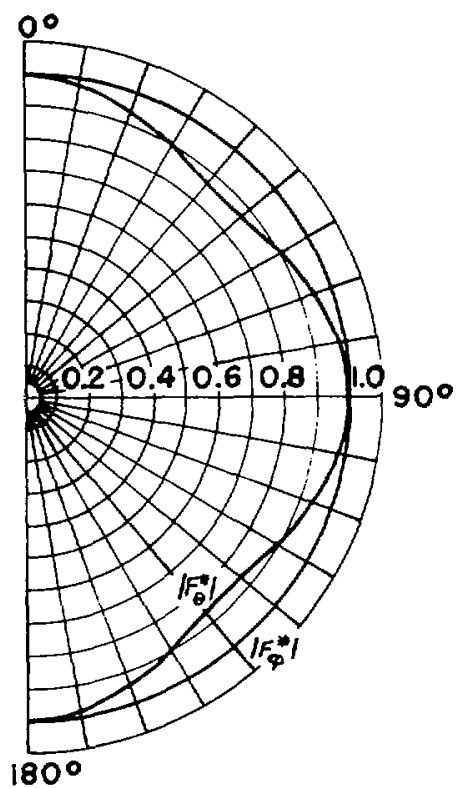

Fig. 20-The electric field radiated by a small homogeneous ring quasi-array of oblique dipoles with $\gamma=\pi / 2$ and $H=1$.

These field components are plotted in Fig. 20. For the polar distances $\theta=0$ degrees, 30 degrees, 60 degrees, and 90 degrees the full-drawn line in Fig. 21 (a)-(d) shows the ellipse which is described by the vector, the electric field strength. 


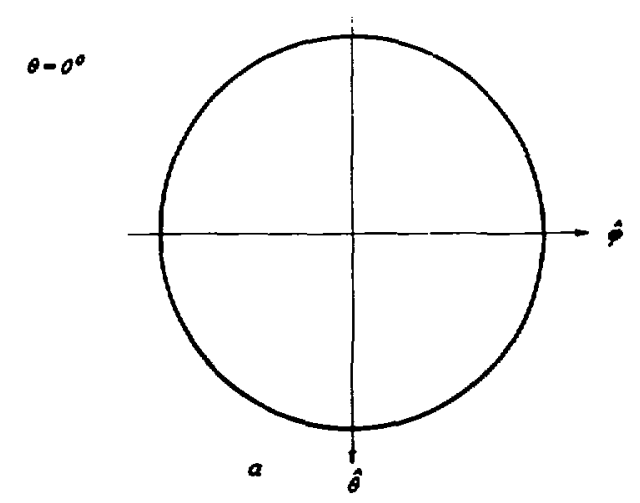

a. $\theta=0^{\circ}$

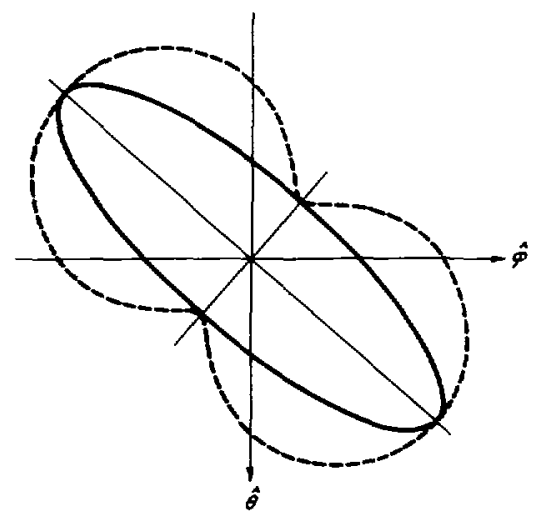

c. $\theta=60^{\circ}$

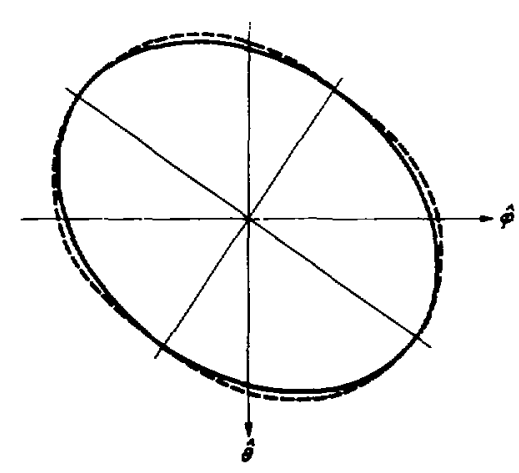

b. $\theta=30^{\circ}$

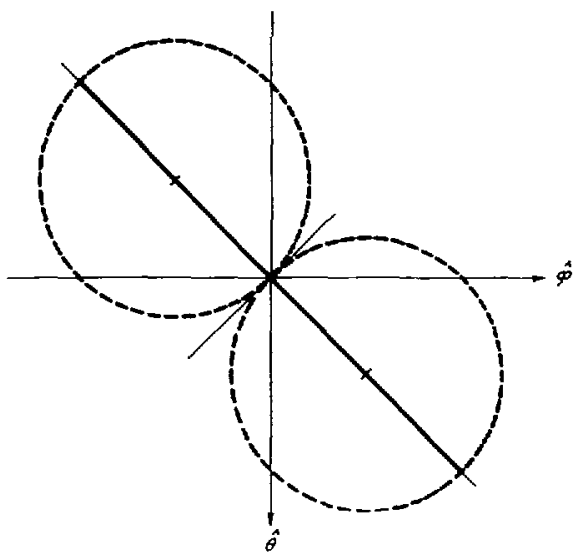

d. $\theta=90^{\circ}$

Fig. 21-The full-drawn lines show the polarization ellipses of the field radiated by a homogeneous ring quasi-array of oblique dipoles with $\gamma=\pi / 2$ and $H=1$ for the pole distances $\theta=0$ degrees, 30 degrees, 60 degrees, and 90 degrees. The dotted lines indicate the voltage induced in a linear antenna placed in this field.

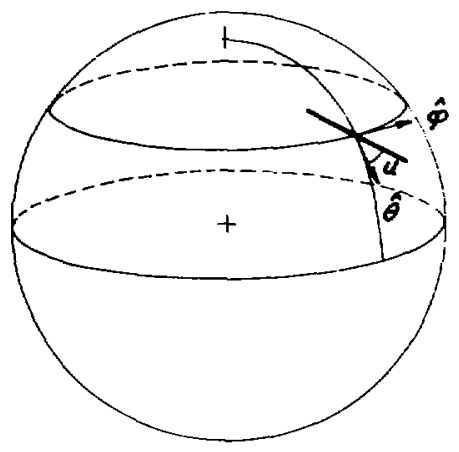

Fig. 22-Linear antenna placed in the direction $(\theta, \phi)$ and oriented in the $(\theta, \phi)$-plane as indicated by the angle $u$.

If in the field of the ring quasi-array placed at the origin we place a linear antenna as shown in Fig. 22, the voltage induced in the linear antenna, will be given as a function of the angle $u$ for various values of the polar distance $\theta$ by the dotted curves in Fig. 21. From this we see that for most placings and orientations of the linear antenna the induced voltage will be different from zero. However, when the linear antenna is placed in the horizontal plane, there will be a certain direction of the antenna for which no voltage is induced in the antenna.

If the sequence of the current phase in the ring quasiarray is inverted; i.e., if we choose $H=-1$ instead of $H=1$, as done above, the diagrams shown in Fig. 21 (a)-(d) will be reflected in the $\theta$-axis. If in any case we choose the sequence of the current phase of the ring quasi-array giving the maximum induced voltage in the linear antenna, we obtain for $\theta=0$ degrees, 30 degrees, 60 degrees, and 90 degrees the induced voltages in the linear antenna given by the curves in Fig. 23(a)-23(d). It appears from this figure that the induced voltage varies only to a small degree with the placing and the orientation of the antenna when reversal of the current phase is applied. 


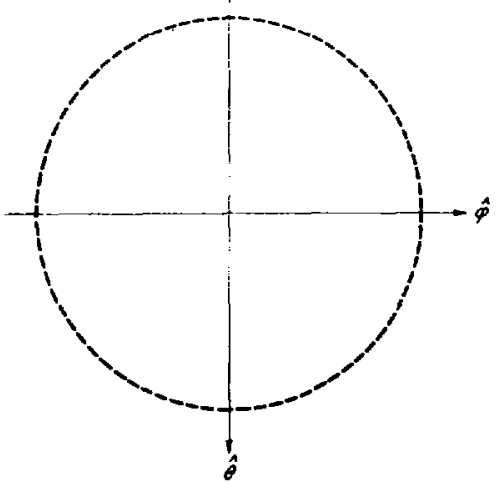

2 $\theta=0^{\circ}$

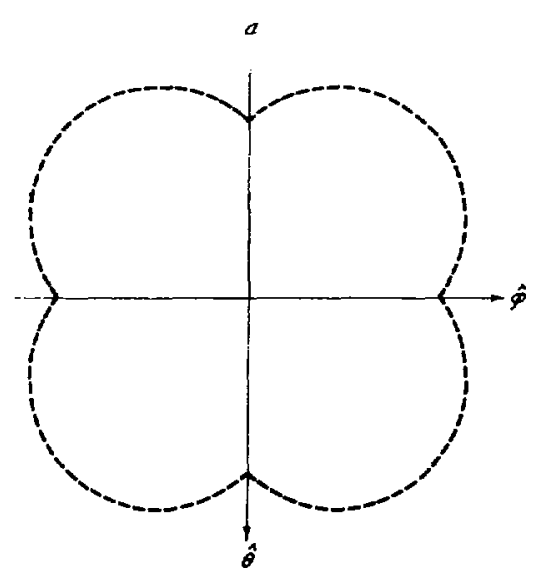

C $\theta=60^{\circ}$

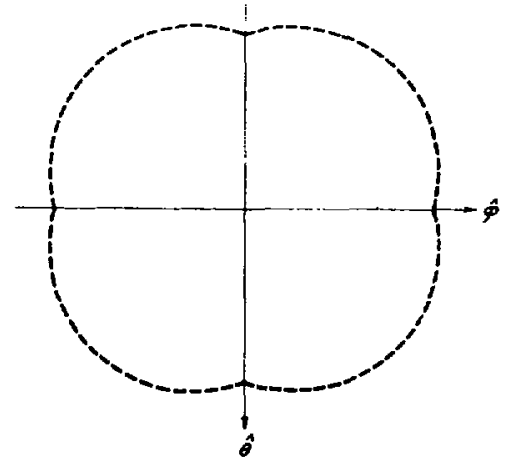

b $\theta=30^{\circ}$

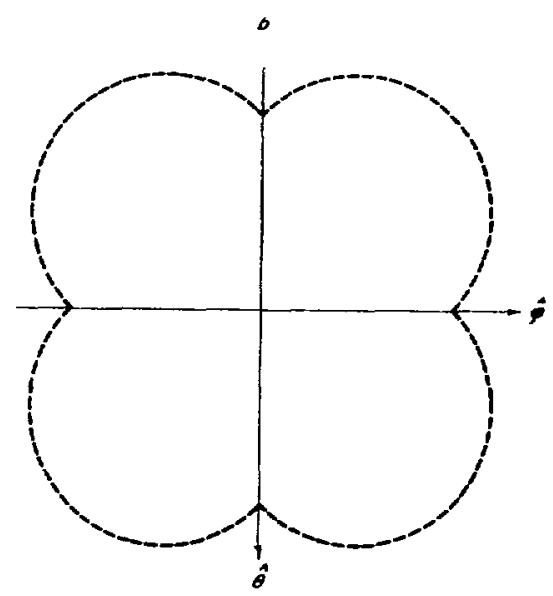

d $\theta=90^{\circ}$

Fig. 23-Induced voltage in a linear antenna placed in the field of a homogeneous ring quasi-array of oblique dipoles with $\gamma=\pi / 2$ and $H=1$, when reversal of the sequence of the current phase is allowed.

As is well known, it is not possible to design an isotropic antenna. ${ }^{28}$ Various attempts have been made to design antennas the properties of which approximate those of an isotropic antenna. However, for all of these antennas it is true that at least for one direction in space towards, and for one orientation of a linear receiving antenna they will induce the voltage zero in this antenna. In applications where reversal of the sequence of the current phase is permissible, the ring quasi-array described here will be a good substitute for an isotropic antenna.

As a practical design of the ring quasi-array described here it will probably be expedient to use a turnstile antenna, the four arms of which are turned upwards so that they form the angle $v$ with the vertical direction, as shown in Fig. 24. The field from such a "quadruped antenna" will to some degree deviate from the field from the ring quasi-array considered above. By a suitable choice of the length of the arms of the antenna

${ }^{28} \mathrm{H}$. F. Mathis, "A short proof that an isotropic antenna is impossible," Proc. IRE, vol. 39, p. 970; August, 1951.

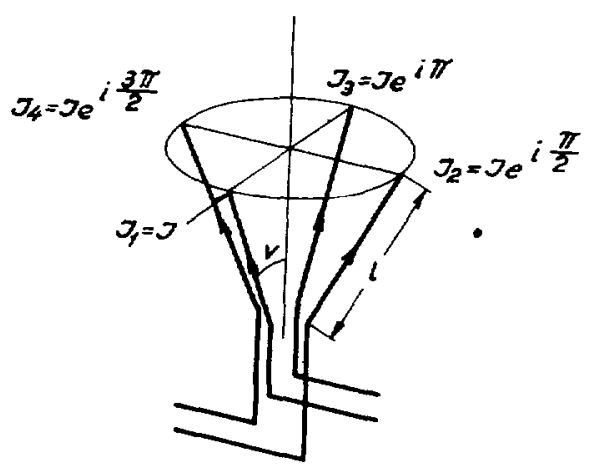

Fig. 24-Quad̦ruped antenna.

and of the angle $v$ it will, however, be possible to obtain a satisfactory radiation diagram.

\section{SUPER-Gain}

In the preceding sections it has been demonstrated that the field radiated from a homogeneous ring array or ring quasi-array will concentrate the more around 
the horizontal plane the larger the increment $H 2 \pi$ of the current phase during one revolution. By making $H$ sufficiently large, it is possible for a homogeneous ring quasi-array of arbitrarily chosen dimensions to obtain an arbitrarily large gain. This result is inconsistent with the practical experience generally made in the antenna theory as this shows that for an antenna of given dimension it is practically impossible to obtain a gain that essentially surpasses the gain for a conventional antenna of the same diameter. Since the early forties, a phenomenon such as the one described here has aroused considerable interest among antenna theorists and has been investigated from various points of view under the name of "super-gain." As for the literature on this subject, reference is made to an earlier paper, ${ }^{7}$ in which a critical investigation of the problem of supergain has been made, and to another paper, ${ }^{8}$ in which the connection between the theory of super-gain and information theory is treated.

In a few words the solution to the problem of supergain may be thus formulated: it is certainly possible to obtain a gain that considerably surpasses the gain that is "natural" to the antenna in question; but as a result the antenna will acquire a $Q$ so large that it will be inapplicable in practice.

For determining the approximate minimum value which a homogeneous ring array or a homogeneous ring quasi-array may have, if super-gain is not to occur, we shall base our considerations on a paper by Woodward and Lawson. ${ }^{29}$ In this paper the authors utilize the fact that the current distribution on a linear antenna array and the array characteristic are Laplace transforms of each other. They hereby demonstrate that in order

${ }^{29}$ P. M. Woodward and J. D. Lawson, "The theoretical precision with which an arbitrary radiation-pattern may be obtained from a source of finite size," Jour. IEE, Pt. III, vol. 95, pp. 363$369 ; 1948$. to avoid super-gain for such an antenna we must demand that the greater part of the produced field is radiated at real, and not at imaginary, angles. Similarly, for the homogeneous ring array dealt with above we must demand that the greater part of the array characteristic $G \sim J_{H}(k a \sin \theta)$ comes within the real domain of $\theta$; i.e., for $t=\sin \theta<1$. Consequently, we must demand that the function $J_{H}(k a t)$ decreases rapidly towards zero for $t>1$. In order that this may apply we must have

$$
a>a_{\mathrm{min}}=\frac{H \lambda}{2 \pi} .
$$

Accordingly, for the smallest usuable antenna array the phase of the current increases just as much per unit of length as for a conventional linear, endfire array.

By a similar argument the same result is obtained for homogeneous ring quasi-arrays of tangential and radial dipoles.

The author would like to mention the following two papers which recently came to his attention. W. Burkhardtsmaier and U. Finkbein, "Kreisgruppenantennen als Rund- und Richtstrahler," Elektrotech., vol. 4, pp. 239-244, 284-290; 1950. R. H. DuHamel, "Pattern synthesis for antenna arrays on circular, elliptical, and spherical surfaces," N6-ori-71, Task XV, ONR Project No. 076161, Tech. Rep. No. 16; 1950.

\section{ACKNOWLEDGMENT}

In the preparation of this paper the author has made use of articles previously published by the author in the Journal of Applied Physics ${ }^{2}$ and in the Proceedings OF THE INSTitute of Radio EngIneERs. ${ }^{3,4}$ I am indebted to The American Institute of Physics, Inc. and The Institute of Radio Engineers, Inc. for permission to use these papers.

The English translation of the paper was revised by Mr. Oscar Kasch, Sworn Translator and Interpreter.

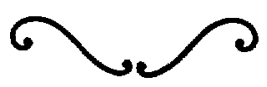

